\title{
MIXED NORM ESTIMATES OF SCHRÖDINGER WAVES AND THEIR APPLICATIONS
}

\author{
MYEONGJU CHAE, YONGGEUN CHO, AND SANGHYUK LEE
}

\begin{abstract}
In this paper we establish mixed norm estimates of interactive Schrödinger waves and apply them to study smoothing properties and global well-posedness of the nonlinear Schrödinger equations with mass critical nonlinearity.
\end{abstract}

\section{INTRODUCTION}

The Sctrichartz estimate shows the dispersive nature of Schrödinger waves, which can be formulated via mixed norm ([20, 13]). More precisely, for admissible $(q, r)$

$$
\left\|e^{i t \Delta} f\right\|_{L_{t}^{q} L_{x}^{r}} \lesssim\|f\|_{L^{2}} .
$$

Here a pair $(q, r)$ is said to be admissible if it satisfies $\frac{2}{q}=n\left(\frac{1}{2}-\frac{1}{r}\right), q, r \geq 2$ with exception $(q, r)=(2, \infty)$ when $n=2$ and $e^{i t \Delta}$ denotes the free propagator of Schrödinger equation.

Due to scaling, the frequency localization via Littewood-Paley decomposition does not give any improvement to the aforementioned Strichartz estimates. However, it was observed by Bourgain [1] that by considering low and high frequency interactions of two Schrödinger waves, namely bilinear control of $e^{i t \Delta} f e^{i t \Delta} g$, it is possible to obtain a refinement of Strichartz estimate in $L_{t, x}^{2}\left(\mathbb{R} \times \mathbb{R}^{2}\right.$ ) (note that $(4,4)$ is an admissible pair when $n=2$ ). In [15] Keranni and Vargas recently extended Bourgian's reults to higher dimensions by showing that a sharp $L_{t, x}^{(n+2) / n}\left(\mathbb{R} \times \mathbb{R}^{n}\right), n \geq 1$ estimate holds for the interactive Schrödinger waves.

Our first result is that such refinements of Strichartz estimates are also valid in the mixed norm setting for $n \geq 3$. Actually it gives stronger interactive estimate which is stated as follows:

2000 Mathematics Subject Classification. 42B25, 35Q40, 35Q55.

Key words and phrases. mixed norm estimates, interactive Schrödinger waves, mass critical nonlinearity, smoothing property, global well-posedness.

Y. Cho is supported in part by the Korea Research Foundation Grant KRF-2008-313-C00065. S. Lee is supported in part by the grant KOSEF-2007-8-1220. 
Theorem 1.1. Let $n \geq 2$. Let $(q, r)$ satisfy that $2 / q=n(1 / 2-1 / r), 2<r<4$, and $q>2$. Then for $|s|<1-2 / r$,

$$
\left\|e^{i t \Delta} f e^{i t \Delta} g\right\|_{L_{t}^{q / 2} L_{x}^{r / 2}} \lesssim\|f\|_{\dot{H}^{s}}\|g\|_{\dot{H}^{-s}} .
$$

The estimate trivially holds for $(r, s)=(2,0)$ by Plancherel's theorem and when $n=2$ it was actually obtained in [15] including $(q, r)=(4,4)$. This estimate obviously has a scaling structure in $L^{2}$ space so that the estimate is invariant along the admissible $(q, r)$. The above estimate makes it possible to move a certain amount of derivative on one to the other function. So it is useful when one studies the smoothing property of nonlinear Schrödingers of power type. The range on $s$ is sharp, since (1.1) fails for $|s|>1-2 / r$ (see the discussion below Proposition 2.1). The estimate (1.1) is strongly connected to the bilinear restriction estimates for the paraboloid (see [15, 17, 21]). In fact, for the proof of Theorem 1.1 we establish estimates for bilinear interactions between waves at different frequency. It relies on the argument used to prove the bilinear restriction estimate for the paraboloid [17, 21], which makes use of wave packet decomposition and induction on scaling (see Proposition 2.1 and Corollary 2.4 below).

Aside from the power type, one of the most typical nonlinearity is that of Hartree in the study of nonlinear Schrödiner equations (see (1.4) below). To handle the Hartree type nonlinearity, we consider the trilinear operator $\mathcal{H}$ which is given by

$$
\mathcal{H}(f, g, h) \equiv|\nabla|^{2-n}\left(e^{i t \Delta} f e^{i t \Delta} g\right) e^{i t \Delta} h
$$

Here $|\nabla|^{2-n}$ is the pseudo-differential operator with symbol $|\xi|^{2-n}$ which is the convolution with $c_{n}|x|^{-2}$. To make the operator have sense, we assume $n \geq 3$ throughout the paper when we use the notation $|\nabla|^{2-n}$. As it is turned out (see Theorem 1.3 and Theorem 1.4), the trilinear estimate enables us to control the interaction of waves arising in Hartree type nonlinearity more effectively. It is stated as follows:

Theorem 1.2. Let $n \geq 3$ and let $(\tilde{q}, \tilde{r})$ be admissible. Suppose that $s_{1}, s_{2}, s_{3}$ are positive numbers satisfying $\sum s_{i}=1$. Then, if $s_{3}>\frac{1}{2}$

$$
\|\mathcal{H}(f, g, \nabla h)\|_{L_{t}^{\tilde{q}^{\prime}} L_{x}^{\tilde{r}^{\prime}}} \lesssim\|f\|_{\dot{H}^{s_{1}}}\|g\|_{\dot{H}^{s_{2}}}\|h\|_{\dot{H}^{s_{3}}} .
$$

If $s_{1}>\frac{1}{2}$, then

$$
\|\mathcal{H}(\nabla f, g, h)\|_{L_{t}^{\tilde{q}^{\prime}} L_{x}^{\tilde{r}^{\prime}}} \lesssim\|f\|_{\dot{H}^{s_{1}}}\|g\|_{\dot{H}^{s_{2}}}\|h\|_{\dot{H}^{s_{3}}} .
$$

It should be noticed that the estimates (1.2), (1.3) are invariant under scaling for all admissible $(\tilde{q}, \tilde{r})$ (cf. Lemma 2.5). For the proof we first show frequency localized estimates (Proposition 2.6 below) which also rely on the bilinear interaction estimates and the scaling structure of $\mathcal{H}$. Compared with (1.1), a stronger interaction 
estimate is possible thanks to operator the $|\nabla|^{2-n}$ which gives additional decay in frequency space.

Now we consider applications of Theorem 1.1 and 1.2 to nonlinear Schrödinger equations. We are concerned with the Cauchy problem of $L^{2}$ critical nonlinear Schrödinger equation in $\mathbb{R}^{n}, n \geq 3$, of which nonlinear part is given by the nonlinear potential $V(u)$ of Hartree or power type:

$$
\left\{\begin{array}{l}
i u_{t}+\Delta u=V(u) u, \quad(t, x) \in[0, T] \times \mathbb{R}^{n}, T>0, \\
u(0, x)=u_{0}(x) \in H^{s}\left(\mathbb{R}^{n}\right) .
\end{array}\right.
$$

That is to say, $V(u)=\kappa|x|^{-2} *|u|^{2}$ or $V(u)=\kappa|u|^{\frac{4}{n}}$ with $\kappa= \pm 1$. Here $u$ : $[0, T] \times \mathbb{R}^{n} \rightarrow \mathbb{C}$ is a complex valued function. If $u$ is a solution to (1.4), the scaled function $\lambda^{\frac{n}{2}} u\left(\lambda^{2} t, \lambda x\right), \lambda>0$ is also a solution. Hence (1.4) is invariant under the scaling in $L^{2}$ space (i.e. $L^{2}$ critical). By the Duhamel's principle the problem (1.4) is equivalent to solving the integral equation for $t \in[0, T]$;

$$
u(t)=e^{i t \Delta} u_{0}-i \int_{0}^{t} e^{i t\left(t-t^{\prime}\right) \Delta}(V(u) u)\left(t^{\prime}\right) d t^{\prime} .
$$

It is well known that the problem (1.4) is locally wellposed for every $s \geq 0$ (see [2, 22]). The lifespan of solution $u$ depends on the $H^{s}$ norm if $u_{0} \in H^{s}, s>0$, and the profile of $u_{0}$ if $u_{0} \in L^{2}$, respectively. The solution $u \in C\left([0, T] ; H^{s}\right)$ to (1.4) satisfies conservation laws, namely, mass and energy; for any $t \in[0, T]$, if $s \geq 0$

$$
\|u(t)\|_{L^{2}}^{2}=\left\|u_{0}\right\|_{L^{2}}^{2}
$$

and if $s \geq 1$

$$
E(u(t)) \equiv \frac{1}{2}\|\nabla u(t)\|_{L^{2}}^{2}+\omega \int V(u(t))|u(t)|^{2} d x=E\left(u_{0}\right),
$$

where $\omega=1 / 4$ if $V(u)=\kappa|x|^{-2} *|u|^{2}$ and $\omega=\frac{n}{4+2 n}$ if $V(u)=\kappa|u|^{\frac{4}{n}}$. If the data is sufficiently smooth $(s \geq 1)$, various results were established by using the classical energy argument. However, it does not work any longer when $0 \leq s<1$ and there has been a lot of works devoted to extending those results to lower regularity initial data (for instance see [1, 6, 9]).

We firstly apply Theorem 1.1, 1.2 to study the smoothing properties of solutions to the Cauchy problem (1.4). We consider a strong global (in $x$-space) smoothing effect such that the Duhamel's part

$$
D(t) \equiv u(t)-e^{i t \Delta} u_{0} \in C\left([0, T] ; H^{1}\right)
$$

for all $T$ within the lifespan when the initial data $u_{0}$ is in $H^{s}, 0 \leq s<1$. The smoothing actually stems from the interaction of Schrödinger waves arising in the nonlinear term. It was first observed by Bourgain [1] for $V(u)=\kappa|u|^{2}, n=2, s>2 / 3$ 
and later extended by Keranni and Vargas [15] for $V(u)=\kappa|u|^{\frac{4}{n}}, n \geq 1, s>s_{n}$, where $s_{1}=3 / 4, s_{n}=n /(n+2)$ for $2 \leq n \leq 4, s_{n}=\left(n^{2}+2 n-8\right) / n(n+2)$. To utilize the interaction, they established refined bilinear Strichartz estimates in $L_{t, x}^{\frac{n+2}{n}}$ as mentioned above. In the following, we get better smoothing effects that (1.6) holds for a rougher $u_{0}$, using the Theorems 1.1 and 1.2 together with the duality arguments based on the Bourgain space ([1, 15]).

Theorem 1.3. Let $n \geq 3$. (1) If $u_{0} \in H^{s}\left(\mathbb{R}^{n}\right)$ and $1 / 2<s<1$, then there is a maximal existence time $T^{*}>0$ such that a unique solution $u$ to (1.4) with $V(u)=\kappa|x|^{-2} *|u|^{2}$ exists in $C\left(\left[0, T^{*}\right) ; H^{s}\right)$ and $D$ satisfies (1.6) for all $T<T^{*}$.

(2) Let $s_{n}=\frac{1}{2}$ for $n=3,4$ and $s_{n}=1-\frac{8}{n^{2}}$ for $n \geq 5$. If $u_{0} \in H^{s}\left(\mathbb{R}^{s}\right), s_{n}<$ $s<1$, then there is a maximal existence time $T^{*}$ such that a unique solution $u$ in $C\left(\left[0, T^{*}\right) ; H^{s}\right)$ to (1.4) with $V(u)=\kappa|u|^{\frac{4}{n}}$ and $D$ satisfies (1.6) for all $T<T^{*}$.

In part (2) we do not have any improvement on 2-d result which was obtained in [15] $\left(s_{2}=\frac{1}{2}\right)$. The above result shows that the Hartree type interaction is more effective than the power type when $n \geq 5$, which may be interpreted as weaker (of lower power) nonlinearity causes a lower interaction between the waves. The smoothing effect can be used to show an $H^{1}$ mechanism for the blowup phenomenon of the Cauchy problem (1.4) (see Remark 1.3 of [15]). In [15], it was shown that if $T^{*}$ is finite, then

$$
\|\nabla D(t)\|_{L^{2}} \gtrsim\left(T^{*}-t\right)^{-1 / 2}
$$

for power type NLS provided that (1.6) holds for all $T<T^{*}$. Hence, part (2) of Theorem 1.3 extends the possible range of $s$. Similarly, using part (1) of Theorem 1.3 and the argument in [15] together with well-known scaling argument, one can also get the same blowup rate of $D(t)$ for the finite time blowup solution of Hartree type NLS as long as $u_{0} \in H^{s}\left(\mathbb{R}^{n}\right)$ and $1 / 2<s<1$.

We now consider the global well-posedness of defocusing $L^{2}$ critical Hartree equation, (1.4) with $\kappa=+1$, for rough initial data in $H^{s}, 0<s<1$. Recently Chae and Kwon [3] considered the same problem (1.4) and they got global well-posedness for $u_{0} \in H^{s}, 2(n-2) /(3 n-4)<s<1$. Their result is based on the so called $I$-method. (For details and recent development of $I$-method, we refer readers to 4, 5, 6, 1, 9, 10, 11, 12.) We here make further improvement. By exploiting the interaction of Schrödinger waves systematically (Proposition 2.6), we obtain better decay estimates for almost energy conservation and interaction Morawetz inequality (see Proposition 4.1, 4.2) which are the major estimates for I-method. As a consequence we get the following global well-posedness theorem.

Theorem 1.4. Let $n \geq 3$ and $V(u)=|x|^{-2} *|u|^{2}$. Then the initial value problem of (1.4) is globally well-posed for data $u_{0} \in H^{s}\left(\mathbb{R}^{n}\right)$ when $\frac{4(n-2)}{7 n-8}<s<1$. 
The global well-posedness for the spherically symmetric data in $L^{2}$ was shown by Miao, Xu and Zhao [18]. They adopted the method due to Killip, Tao and Visan [14]. For the 2-d cubic NLS, Colliander and Roy [9] recently combined the improved estimate in [7] with a Mowawetz error estimate by using the double layer bootstrap in time, and established the global well-posedness for the $L^{2}$ critical NLS on $\mathbb{R}^{2}$ with data in $H^{s}, s>1 / 3$. It seems highly possible that such approach also makes further progress for the Hartree equations if it is combined with the results of this paper. We hope to address such issues somewhere else. Compared to the previous works, our proof of almost energy conservation and interaction Morawetz inequality is more systematic and flexible. We believe that it may be useful in studies of various related problems.

This paper is organized as follows: In Section 2 we will obtain the bilinear interaction estimate, trilinear Hartree type interaction estimate, and prove Theorem 1.1, 1.2. In Section 3 we will show the local well-posedness and smoothing effect of Duhamel's part of solutions to (1.4). The Section 4 is devoted to showing the global well-posedness of defocusing Hartree equation. Lastly we append a brief introduction to wave packet decomposition of Schrödinger wave, which will be used in Section 2.

We now list the notations which are frequently used in the paper:

- $A \lesssim B$ means that $A \leq C B$ for some constant $C>0$ which may vary from lines to lines. We also write $A \sim B$ when $A \lesssim B$ and $B \lesssim A$.

- The symbol $\nabla$ denotes the gradient $\left(\partial / \partial_{1}, \cdots, \partial / \partial_{n}\right)$ and $\Delta$ the Laplacian $\nabla \cdot \nabla=$ $\sum_{j} \partial^{2} / \partial_{j}^{2}$. We also denote $(-\Delta)^{\frac{1}{2}}$ by $|\nabla|$.

- Let $J_{T}$ be the time interval $[0, T]$. For a measurable function $F$ the mixed norm is defined by $\|F\|_{L_{t}^{q} L_{x}^{r}\left(J_{T} \times \mathbb{R}^{n}\right)}=\left(\int_{J_{T}}\left(\int_{\mathbb{R}^{n}}|F(t, x)|^{r} d x\right)^{\frac{q}{r}}\right)^{\frac{1}{q}}$. We use $\|F\|_{L_{t}^{q} L_{x}^{r}}$ to denote $\|F\|_{L_{t, x}^{q}\left(\mathbb{R} \times \mathbb{R}^{n}\right)}$. $L^{p}$ is the usual Lebesgue space $L_{x}^{p}\left(\mathbb{R}^{n}\right)$.

- The Fourier transform of $f$ is defined by $\mathcal{F}(f)(\xi)=\widehat{f}(\xi) \equiv \int e^{-i x \cdot \xi} f(x) d x$ and its inverse by $\mathcal{F}^{-1}(g)(x) \equiv(2 \pi)^{-n} \int e^{i x \cdot \xi} g(\xi) d \xi$. Hence $e^{i t \Delta} f(x)=\mathcal{F}^{-1}\left(e^{-i t|\cdot|^{2}} \mathcal{F}(f)\right)(x)$ $=(2 \pi)^{-n} \int_{\mathbb{R}^{n}} e^{i\left(x \cdot \xi-t|\xi|^{2}\right)} \widehat{f}(\xi) d \xi$.

- Let $N$ denote dyadic number and let $P_{N}$ be the Littlewood-Paley projection operator with symbol $\chi(\xi / N) \in C_{0}^{\infty}$ supported in the annulus $A(N)=\{1 / 2 N \leq|\xi| \leq$ $2 N\}$ such that $\sum_{N} P_{N}=i d$. We also define $\widetilde{P}_{1}=i d-\sum_{N>1} P_{N}$.

- The inhomogeneous Sobolev space $H^{s}\left(=H^{s}\left(\mathbb{R}^{n}\right), s \in \mathbb{R}\right)$ denotes the space $\{f \in$ $\left.\mathcal{S}^{\prime}:\|f\|_{H^{s}}<\infty\right\}$, where $\|f\|_{H^{s}} \equiv\left(\sum_{N \geq 0} N^{2 s}\left\|P_{N} f\right\|_{L^{2}}^{2}+\|f\|_{2}^{2}\right)^{\frac{1}{2}} \sim\left\|\langle\nabla\rangle^{s} f\right\|_{L^{2}}=$ $\left(\int\langle\xi\rangle^{2 s}|\widehat{f}(\xi)|^{2} d \xi\right)^{\frac{1}{2}}$. Here $\langle A\rangle=\sqrt{1+|A|^{2}}$. We will also use the homogeneous 
Sobolev space $\dot{H}^{s}=\left\{f \in \mathcal{S}^{\prime} / \mathcal{P}:\|f\|_{\dot{H} s}<\infty\right\}$, where $\mathcal{P}$ is the totality of polynomials and the seminorm $\|f\|_{\dot{H}^{s}} \equiv\left(\sum_{N \text { :dyadic }} N^{2 s}\left\|P_{N} f\right\|_{L^{2}}^{2}\right)^{\frac{1}{2}} \sim\left\||\nabla|^{s} f\right\|_{L^{2}}$. We note here that if $|s|<n / 2$, the definition of $\dot{H}^{s}$ makes sense in $\mathcal{S}^{\prime}$ and $C_{0}^{\infty}$ is dense in $\dot{H}^{s}$ (cf. [19]).

\section{Mixed norm interaction estimates for the Schrödinger WAVES}

In this section we prove first bilinear interaction estimates for the Schrödinger waves. Considering the mixed norm space, it is possible to get a better interaction estimate than the one obtained in [15]. We denote by $B(\xi, \rho)$ the ball centered at $\xi$ with radius $\rho$.

Proposition 2.1. Let $n \geq 2$. Suppose that supp $\widehat{f} \subset B\left(\xi_{0}, \rho_{1}\right)$ and supp $\widehat{g} \subset$ $B\left(\eta_{0}, \rho_{2}\right)$ for some $\left|\xi_{0}\right|,\left|\eta_{0}\right| \leq 1$. If $\left|\xi_{0}-\eta_{0}\right| \sim 1$ and $0<\rho_{1}, \rho_{2} \ll 1$, then for $\epsilon>0$ and $(q, r)$ satisfying that $r \leq 4,2<q$ and $1-\frac{2}{r} \leq \frac{2}{q}<(n+1)\left(\frac{1}{2}-\frac{1}{r}\right)$

$$
\left\|e^{i t \Delta} f e^{i t \Delta} g\right\|_{L_{t}^{q / 2} L_{x}^{r / 2}} \lesssim \min \left(\rho_{1}, \rho_{2}\right)^{\alpha(q, r)-\epsilon}\|f\|_{L^{2}}\|g\|_{L^{2}}
$$

where $\alpha(q, r)=(n+1)(1-2 / r)-4 / q$.

It can be shown that the bounds in the above estimates are sharp up to $\epsilon$. Indeed, assuming $\rho_{1} \leq \rho_{2}$, let us consider the functions $f$ and $g$ given by $\widehat{f}=\chi_{A}$ and $\widehat{g}=\chi_{B}$ for $A=\left\{\xi:\left|\xi_{1}-1\right| \leq \rho_{1}^{2},\left|\xi_{i}\right| \leq \rho_{1}, i=2, \ldots, n\right\}$ and $B=\left\{\xi:\left|\xi_{1}+1\right| \leq\right.$ $\left.\rho_{1}^{2},\left|\xi_{i}\right| \leq \rho_{1}, i=2, \ldots, n\right\}$. Then it is easy to see that $\left|e^{i t \Delta} f(x)\right|,\left|e^{i t \Delta} g(x)\right| \geq c \rho_{1}^{n+1}$ if $\left|x_{1}\right|,|t| \leq c \rho_{1}^{-2}$ and $\left|x_{i}\right| \leq c \rho_{1}^{-1}$ for some $c>0, i=2, \ldots, n$. Hence

$$
\rho_{1}^{n+1-\frac{2(n+1)}{r}-\frac{4}{q}} \lesssim \frac{\left\|e^{i t \Delta} f e^{i t \Delta} g\right\|_{L_{t}^{q / 2} L_{x}^{r / 2}}}{\|f\|_{L^{2}}\|g\|_{L^{2}}} .
$$

Letting $\rho_{1} \rightarrow 0$ we can see that this implies $\alpha(q, r)-\epsilon \leq n+1-\frac{2(n+1)}{r}-\frac{4}{q}$ for any $\epsilon>0$. It also shows the failure of the estimates when $\frac{2}{q}>(n+1)\left(\frac{1}{2}-\frac{1}{r}\right)$. The example above is actually the squashed cap function which was used to show the sharpness of bilinear restriction estimates [23].

Remark 2.2. Using (2.2) below and Plancherel's theorem, we can show that for $(q, r)$ satisfying $1-2 / r \geq 2 / q$ and $r \leq 4$,

$$
\left\|e^{i t \Delta} f e^{i t \Delta} g\right\|_{L_{t}^{q / 2} L_{x}^{r / 2}} \lesssim \min \left(\rho_{1}, \rho_{2}\right)^{n\left(1-\frac{2}{r}\right)-\frac{2}{q}}\|f\|_{L^{2}}\|g\|_{L^{2}} .
$$

It is also sharp as it can be shown by using the functions $f$ and $g$ with $\widehat{f}=\chi_{A}$ and $\widehat{g}=\chi_{B}$ for $A=\left\{\xi:\left|\xi-e_{1}\right| \leq \rho_{1}\right\}$ and $B=\left\{\xi:\left|\xi_{1}+e_{1}\right| \leq \rho_{1}\right\}$

For the proof of Proposition 2.1 we will use the wave packet decomposition for the Schrödinger operator. Such decomposition was used to study Fourier restriction estimates [17, 21, 25]. 
Proof of Proposition 2.1. By symmetry we may assume $\rho_{1} \leq \rho_{2}$. We start with recalling the estimates

$$
\left\|e^{i t \Delta} f e^{i t \Delta} g\right\|_{L_{t}^{q / 2} L_{x}^{r / 2}} \lesssim\|f\|_{L^{2}}\|g\|_{L^{2}}
$$

for $\frac{2}{q}<(n+1)\left(\frac{1}{2}-\frac{1}{r}\right), 2<q, r \leq 4$. See Theorem 2.3 of [17]. Also we make use of the estimate

$$
\left\|e^{i t \Delta} f e^{i t \Delta} g\right\|_{L_{t}^{2} L_{x}^{2}} \lesssim \rho_{1}^{\frac{n-1}{2}}\|f\|_{L^{2}}\|g\|_{L^{2}} *
$$

which already appeared in several literatures (for instance see [1] and [15]). For the convenience of reader we give a simple proof based on Plancherel's theorem.

Using an affine transformation we may assume $\xi_{0}=0$. By decomposing the Fourier support of $g$ into finite number of sets, rotation and dilation, it is enough to show (2.2) whenever $f$ and $g$ are Fourier-supported in $B\left(0, \rho_{1}\right)$ and $B\left(e_{1}, \delta\right)$ for some $0<\delta \ll 1$, respectively. We write

$$
e^{i t \Delta} f(x) e^{i t \Delta} g(x)=\int e^{i\left(x \cdot(\xi+\eta)-t\left(|\xi|^{2}+|\eta|^{2}\right)\right)} \widehat{f}(\xi) \widehat{g}(\eta) d \xi d \eta
$$

Freezing $\bar{\xi}=\left(\xi_{2}, \ldots, \xi_{n}\right)$, we consider a bilinear operator

$$
B_{\bar{\xi}}(f, g)=\int e^{i\left(x \cdot(\xi+\eta)-t\left(|\xi|^{2}+|\eta|^{2}\right)\right)} \widehat{f}\left(\xi_{1}, \bar{\xi}\right) \widehat{g}(\eta) d \xi_{1} d \eta .
$$

We make the change of variables $\zeta=\left(\zeta_{1}, \zeta_{2}, \ldots, \zeta_{n+1}\right)=\left(\xi+\eta,|\xi|^{2}+|\eta|^{2}\right)$. Then by direct computation one can see that $\left|\frac{\partial \zeta}{\partial\left(\xi, \eta_{1}\right)}\right|=2\left|\xi_{1}-\eta_{1}\right| \sim 1$ on the supports of $\widehat{f}$ and $\widehat{g}$. Hence making the change of variables $\left(\xi_{1}, \eta\right) \rightarrow \zeta$, applying Plancherel's theorem and reversing the change variables $\left(\zeta \rightarrow\left(\xi_{1}, \eta\right)\right)$, we have

$$
\left\|B_{\bar{\eta}}(f, g)\right\|_{L_{t}^{2} L_{x}^{2}} \lesssim\left\|\widehat{f}\left(\xi_{1}, \bar{\xi}\right) \widehat{g}(\eta)\right\|_{L_{\xi_{1}, \eta}^{2}} .
$$

Since $e^{i t \Delta} f(x) e^{i t \Delta} g(x)=\int B_{\bar{\xi}}(\hat{f}(\cdot, \bar{\xi}), \hat{g}(\cdot)) d \bar{\xi}$, by Minkowski's inequality we get

$$
\left\|e^{i t \Delta} f e^{i t \Delta} g\right\|_{L_{t}^{2} L_{x}^{2}} \lesssim \int\left\|B_{\bar{\xi}}(\hat{f}(\cdot, \bar{\xi}) \hat{g}(\cdot))\right\|_{L_{\xi_{1}, \eta}^{2}} d \bar{\xi} .
$$

This gives the desired estimate (2.2) by Cauchy-Schwarz inequality because $|\bar{\xi}| \leq \rho_{1}$.

When $n=2,3$ we only need to interpolate (2.1), (2.2) and $\left\|e^{i t \Delta} f e^{i t \Delta} g\right\|_{L_{t}^{\infty} L_{x}^{1}} \lesssim$ $\|f\|_{2}\|g\|_{2}$ for the proof of the theorem. It gives all the desired estimates. Hence, similarly when $n \geq 4$, it is sufficient to show that for $\epsilon>0$

$$
\left\|e^{i t \Delta} f e^{i t \Delta} g\right\|_{L_{t}^{q_{\epsilon} / 2} L_{x}^{r_{\epsilon} / 2}} \lesssim \rho_{1}^{\frac{n-3}{2}-\epsilon}\|f\|_{L^{2}}\|g\|_{L^{2}}
$$

Here $\left(q_{\epsilon}, r_{\epsilon}\right)$ converges to $(2,4)$ as $\epsilon \rightarrow 0$. A similar estimate already appeared in [17] for the wave operator and its proof is based on the induction on scale argument. We also follow the same lines of argument. 
Let $\lambda$ be a large number so that $\lambda \gg \rho_{1}^{-\frac{1}{2}}$ and let us set $\mathcal{Q}(\lambda)=Q(\lambda) \times(-\lambda, \lambda)$, where $Q(\lambda)$ is the cube centered at the origin with side length $2 \lambda$. We make an assumption that

$$
\left\|e^{i t \Delta} f e^{i t \Delta} g\right\|_{L_{t}^{1} L_{x}^{2}(\mathcal{Q}(\lambda))} \lesssim \rho_{1}^{\frac{n-3}{2}} \lambda^{\alpha}\|f\|_{L^{2}}\|g\|_{L^{2}}
$$

Due to (2.2) and Hölder's inequality the above is valid with $\alpha=1 / 2$. Now we attempt to suppress $\alpha$ as small as possible.

Let $\{b\}$ be the collection of the $\lambda^{1-\delta}$-cubes $b$ partitioning $\mathcal{Q}(\lambda)$. We make use of the wave packet decomposition and Lemma 4.7 which had crucial role in the proof of the sharp bilinear restriction estimates for the paraboloids [21]. We provided some basic properties of wave packets in the appendix. Using wave packet decomposition at scale $\lambda$ and the triangle inequality, we have

$$
\left\|e^{i t \Delta} f e^{i t \Delta} g\right\|_{L_{t}^{1} L_{x}^{2}(\mathcal{Q}(\lambda))} \leq \sum_{b}\left\|\sum_{T, T^{\prime}} e^{i t \Delta} f_{T} e^{i t \Delta} g_{T^{\prime}}\right\|_{L_{t}^{1} L_{x}^{2}(b)} .
$$

Using the relation $\sim$, we break the mixed integration over $b$ so that

$$
\left\|e^{i t \Delta} f e^{i t \Delta} g\right\|_{L_{t}^{1} L_{x}^{2}(\mathcal{Q}(\lambda))} \leq I+I I
$$

where

$$
\begin{aligned}
I & =\sum_{b}\left\|\sum_{T \sim b \text { and } T^{\prime} \sim b} e^{i t \Delta} f_{T} e^{i t \Delta} g_{T^{\prime}}\right\|_{L_{t}^{1} L_{x}^{2}(b)}, \\
I I & =\sum_{b}\left\|\sum_{T \not b \text { or } T^{\prime} \nsim b} e^{i t \Delta} f_{T} e^{i t \Delta} g_{T^{\prime}}\right\|_{L_{t}^{1} L_{x}^{2}(b)} .
\end{aligned}
$$

For the first we use the induction assumption (2.4) to get

$$
I \leq C \rho_{1}^{\frac{n-3}{2}} \lambda^{\alpha(1-\delta)} \sum_{b}\left\|\sum_{T \sim b} f_{T}\right\|_{L^{2}}\left\|\sum_{T \sim b} g_{T}\right\|_{L^{2}}
$$

because $b$ is a cube of size $\sim \lambda^{1-\delta}$. Hence by (4.23) and Schwarz's inequality

$$
I \leq C \rho_{1}^{\frac{n-3}{2}} \lambda^{\epsilon} \lambda^{\alpha(1-\delta)}\|f\|_{L^{2}}\|g\|_{L^{2}} .
$$

Hölder's inequality and (4.24) give

$$
\left\|\sum_{T \not b \text { or } T^{\prime} \not b} e^{i t \Delta} f_{T} e^{i t \Delta} g_{T^{\prime}}\right\|_{L_{t}^{1} L_{x}^{2}(b)} \leq C \lambda^{c \delta-(n-3) / 4}\|f\|_{L^{2}}\|g\|_{L^{2}} .
$$

Since there are only $\lambda^{c \delta}$-cubes $b$ and $\rho_{1}^{2} \gg \lambda^{-1}$, it follows that

$$
I I \lesssim \rho_{1}^{\frac{n-3}{2}} \lambda^{\epsilon} \lambda^{c \delta}\|f\|_{L^{2}}\|g\|_{L^{2}}
$$

Combining two estimates for $I$ and $I I$, we get

$$
\left\|e^{i t \Delta} f e^{i t \Delta} g\right\|_{L_{t}^{1} L_{x}^{2}(\mathcal{Q}(\lambda))} \lesssim \rho_{1}^{\frac{n-3}{2}}\left(\lambda^{(1-\delta) \alpha}+\lambda^{\epsilon} \lambda^{c \delta}\right)\|f\|_{L^{2}}\|g\|_{L^{2}} .
$$


Therefore we see that the assumption (2.4) implies the above estimate (2.5). Since $\epsilon, \delta>0$ can be chosen to be arbitrarily small, we get for any $\alpha>0$

$$
\left\|e^{i t \Delta} f e^{i t \Delta} g\right\|_{L_{t}^{1} L_{x}^{2}(\mathcal{Q}(\lambda))} \lesssim \rho_{1}^{\frac{n-3}{2}} \lambda^{\alpha}\|f\|_{L^{2}}\|g\|_{L^{2}}
$$

by iterating this implication (2.4) $\rightarrow(2.5)$ finitely many timest. To ungrade this to the global one, we need the following globalization lemma in [17].

Lemma 2.3. Let $S_{1}$ and $S_{2}$ be compact surfaces with boundary $S_{i}=\left\{\left(\xi, \phi_{i}(\xi)\right)\right.$ : $\left.\xi \in U_{i}\right\}$ and the induced Lebesgue measures $d \sigma_{i}(\xi)=d \xi, i=1,2$, which satisfy $\left\|d \sigma_{i}\right\| \lesssim_{i}, \sigma_{i}(B(z, \rho)) \lesssim \rho^{n-1}$ for any $z, \rho>0$ and $\left|\widehat{d \sigma}_{i}(x, t)\right| \leq C_{i}(1+|x|+|t|)^{-\sigma}$ for some $C_{i} \geq 1$ and $\sigma>0$. Suppose that for some $\frac{2+2 \sigma}{\sigma} \geq q_{0}, r_{0} \geq 1$ and $0<\epsilon \ll \sigma$,

$$
\left\|\prod_{i=1}^{2} \widehat{f_{i} d \sigma_{i}}\right\|_{L_{t}^{q_{0}} L_{x}^{r_{0}(\mathcal{Q}(\lambda))}} \leq C_{0} \lambda^{\epsilon} \prod_{i=1}^{2}\left\|f_{i}\right\|_{L^{2}\left(d \sigma_{i}\right)}
$$

Let $\frac{1}{q_{1}}=\frac{1}{q_{0}}-\frac{2 \epsilon}{2 \epsilon+\sigma}\left(\frac{1}{q_{0}}-\frac{\sigma}{2(\sigma+1)}\right), \frac{1}{r_{1}}=\frac{1}{r_{0}}-\frac{2 \epsilon}{2 \epsilon+\sigma}\left(\frac{1}{r_{0}}-\frac{\sigma}{2(\sigma+1)}\right)$. Then, for $q>q_{1}$

$$
\left\|\prod_{i=1}^{2} \widehat{f_{i} d \sigma_{i}}\right\|_{L_{t}^{q} L_{x}^{r_{1}}} \lesssim C_{0}^{1-\frac{\epsilon}{\sigma}}\left(\max \left(C_{1}, C_{2}\right)\right)^{a \epsilon+\left(1-\frac{q_{1}}{q}\right)\left(1-\frac{1}{r_{1}}\right)} \prod_{i=1}^{2}\left\|f_{i}\right\|_{L^{2}\left(d \sigma_{i}\right)}
$$

with some $a>0$ depending on $\sigma$.

Let us define two extension operators by

$$
\widehat{h d \sigma_{1}}=\int e^{i\left(x \cdot \xi-t|\xi|^{2}\right)} \beta\left(\frac{\xi-\xi_{0}}{\rho_{1}}\right) h(\xi) d \xi, \quad \widehat{h d \sigma_{2}}=\int e^{i\left(x \cdot \xi-t|\xi|^{2}\right)} \beta\left(\frac{\xi-\eta_{0}}{\rho_{2}}\right) h(\xi) d \xi
$$

for smooth $\beta$ supported in $B(0,2)$ and $\beta=1$ on $B(0,1)$. Since supp $\widehat{f} \subset B\left(\xi_{0}, \rho_{1}\right)$ and supp $\widehat{g} \subset B\left(\eta_{0}, \rho_{2}\right)$, by Plancherel's theorem it is sufficient to show that the estimate

$$
\left\|\prod_{i=1}^{2} \widehat{h_{i} d \sigma_{i}}\right\|_{L_{t}^{1} L_{x}^{2}(\mathcal{Q}(\lambda))} \lesssim \rho_{1}^{\frac{n-3}{2}} \lambda^{\alpha} \prod_{i=1}^{2}\left\|h_{i}\right\|_{2}
$$

implies the global estimate

$$
\left\|\prod_{i=1}^{2}{\widehat{h_{i} d \sigma_{i}}}_{L_{t}^{q(\alpha) / 2} L_{x}^{r(\alpha) / 2}} \lesssim \rho_{1}^{\frac{n-3}{2}-\epsilon(\alpha)} \prod_{i=1}^{2}\right\| h_{i} \|_{2}
$$

with $q(\alpha) \rightarrow 1, r(\alpha) \rightarrow 2$ and $\epsilon(\alpha) \rightarrow 0$ as $\alpha \rightarrow 0$. Hence using Lemma 2.3, we only need to check that

$$
\left|\widehat{d \sigma}_{i}(x, t)\right| \lesssim(1+|x|+|t|)^{-\frac{n}{2}}
$$

This is easy to see by using stationary phase method because $\rho_{1}, \rho_{2} \ll 1$. It completes the proof of Proposition 2.1.

${ }^{\dagger}$ For this one should note that the constant $c$ in (2.5) is independent of $\epsilon, \lambda$. 
2.1. Proof of Theorem 1.1: Bilinear interaction estimates. We note that the bilinear estimate in Proposition 2.1 is invariant under rescaling when $2 / q=$ $n(1 / 2-1 / r)$. Hence, by Proposition 2.1 and rescaling it is easy to see the following Corollary 2.4, which shows that there is an interactive compensation when one considers the Schrödinger waves of different frequency levels. Throughout the paper we denote by $A(\rho)$ the set $\{\xi:|\xi| \sim \rho\}$.

Corollary 2.4. Let $n \geq 2$. Let $(q, r)$ satisfy that $2 / q=n(1 / 2-1 / r), 2 \leq r \leq 4$. If supp $\widehat{f} \subset A\left(N_{1}\right)$ and supp $\widehat{g} \subset A\left(N_{2}\right)$ for $0<N_{1} \leq N_{2}$, then for any $\epsilon>0$,

$$
\left\|e^{i t \Delta} f e^{i t \Delta} g\right\|_{L_{t}^{q / 2} L_{x}^{r / 2}} \lesssim\left(\frac{N_{1}}{N_{2}}\right)^{1-2 / r-\epsilon}\|f\|_{L^{2}}\|g\|_{L^{2}}
$$

We now give the proof of the Theorem 1.1. The assertion for $s=0$ follows from the Hölder's inequality and Strichartz estimate. By symmetry we may assume that $s>0$. Let $P_{N}$ be the Littlewood-Paley projection as stated in the introduction. For simplicity we set $f_{N}=P_{N} f$ and break $e^{i t \Delta} f e^{i t \Delta} g$ so that

$$
e^{i t \Delta} f e^{i t \Delta} g=\sum_{N_{1}, N_{2}: \text { dyadic }} e^{i t \Delta} f_{N_{1}}\left(e^{i t \Delta} g_{N_{2}}\right) \text {. }
$$

for any $f \in \dot{H}^{s}$ and $g \in \dot{H}^{-s}$. Since $|\nabla| \sim N_{2}$ on the Fourier support of $g_{N_{2}}$, it is enough to show that

$$
\left\|\sum_{N_{1}, N_{2}} e^{i t \Delta} f_{N_{1}} e^{i t \Delta} g_{N_{2}}\right\|_{L_{t}^{q / 2} L_{x}^{r / 2}} \lesssim\left(\sum_{N_{1}} N_{1}^{2 s}\left\|f_{N_{1}}\right\|_{L^{2}}^{2}\right)^{\frac{1}{2}}\left(\sum_{N_{2}} N_{2}^{-2 s}\left\|g_{N_{2}}\right\|_{L^{2}}^{2}\right)^{\frac{1}{2}} .
$$

Let us set $N_{12}=N_{1} N_{2}$. By the triangle inequality

$$
\left\|\sum_{N_{1}, N_{2}} e^{i t \Delta} f_{N_{1}} e^{i t \Delta} g_{N_{2}}\right\|_{L_{t}^{q / 2} L_{x}^{r / 2}} \leq I+I I
$$

where

$$
I=\left\|\sum_{N_{1} \geq 1} \sum_{N_{2}} e^{i t \Delta} f_{N_{12}} e^{i t \Delta} g_{N_{2}}\right\|_{L_{t}^{q / 2} L_{x}^{r / 2}}, \quad I I=\left\|\sum_{N_{1}<1} \sum_{N_{2}} e^{i t \Delta} f_{N_{12}} e^{i t \Delta} g_{N_{2}}\right\|_{L_{t}^{q / 2} L_{x}^{r / 2}} .
$$

Since $I \lesssim \sum_{N_{1} \geq 1} N_{1}^{2 / r-1+\epsilon} \sum_{N_{2}}\left\|f_{N_{12}}\right\|_{L^{2}}\left\|g_{N_{2}}\right\|_{L^{2}}$ by the triangle inequality and Corollary 2.4, we see that

$$
\begin{aligned}
I & \lesssim \sum_{N_{1} \geq 1} N_{1}^{2 / r-1-s+\epsilon} \sum_{N_{2}}\left(N_{12}\right)^{s}\left\|f_{N_{12}}\right\|_{L^{2}} N_{2}^{-s}\left\|g_{N_{2}}\right\|_{L^{2}} \\
& \lesssim \sum_{N_{1} \geq 1} N_{1}^{2 / r-1-s+\epsilon}\left(\sum_{N_{1}} N_{1}^{2 s}\left\|f_{N_{1}}\right\|_{L^{2}}^{2}\right)^{\frac{1}{2}}\left(\sum_{N_{2}} N_{2}^{-2 s}\left\|g_{N_{2}}\right\|_{L^{2}}^{2}\right)^{\frac{1}{2}} \\
& \lesssim\left(\sum_{N_{1}} N_{1}^{2 s}\left\|f_{N_{1}}\right\|_{L^{2}}^{2}\right)^{\frac{1}{2}}\left(\sum_{N_{2}} N_{2}^{-2 s}\left\|g_{N_{2}}\right\|_{L^{2}}^{2}\right)^{\frac{1}{2}}
\end{aligned}
$$


provided $2 / r-1-s+\epsilon<0$. We now turn to $I I$. By the triangle inequality and Corollary 2.4, $I I \lesssim \sum_{N_{1}<1} N_{1}^{1-2 / r-\epsilon} \sum_{N_{2}}\left\|f_{N_{12}}\right\|_{L^{2}}\left\|g_{N_{2}}\right\|_{L^{2}}$. Hence, by Schwarz's inequality

$$
\begin{aligned}
I I & \lesssim \sum_{N_{1}<1} N_{1}^{1-2 / r-\epsilon-s}\left(\sum_{N_{1}} N_{1}^{2 s}\left\|f_{N_{1}}\right\|_{L^{2}}^{2}\right)^{\frac{1}{2}}\left(\sum_{N_{2}} N_{2}^{-2 s}\left\|g_{N_{2}}\right\|_{L^{2}}^{2}\right)^{\frac{1}{2}} \\
& \lesssim\left(\sum_{N_{1}} N_{1}^{2 s}\left\|f_{N_{1}}\right\|_{L^{2}}^{2}\right)^{\frac{1}{2}}\left(\sum_{N_{2}} N_{2}^{2(1-s)}\left\|g_{N_{2}}\right\|_{L^{2}}^{2}\right)^{\frac{1}{2}}
\end{aligned}
$$

as long as $1-2 / r-\epsilon-s>0$. This completes the proof of Theorem 1.1.

\subsection{Proof of Theorem 1.2; Trilinear interaction of Hartree type nonlin-}

earity. First we recall the following which is a consequence of Strichartz estimate and Hardy-Littlewood -Sobolev inequality.

Lemma 2.5. For any admissible $(\tilde{q}, \tilde{r})$,

$$
\|\mathcal{H}(f, g, h)\|_{L_{t}^{\tilde{q}^{\prime}} L_{x}^{\tilde{r}^{\prime}}} \lesssim\|f\|_{L^{2}}\|g\|_{L^{2}}\|h\|_{L^{2}}
$$

and the estimates are invariant under the rescaling $(f, g, h) \rightarrow\left(f_{\lambda}, g_{\lambda}, h_{\lambda}\right)=\left(\lambda^{\frac{n}{2}} f(\lambda \cdot)\right.$, $\left.\lambda^{\frac{n}{2}} g(\lambda \cdot), \lambda^{\frac{n}{2}} h(\lambda \cdot)\right)$ for any $\lambda>0$.

To show this, observe that for any admissible $(\tilde{q}, \tilde{r})$ there is an admissible $(q, r)$ such that $\left(1 / \tilde{q}^{\prime}, 1 / \tilde{r}^{\prime}\right)+(0,(n-2) / n)=3(1 / q, 1 / r)$. Then, using Hölder's and Hardy-Littlewood -Sobolev inequalities one can get the desired estimate.

Via frequency localization on annulus we first obtain the following trilinear interaction estimate.

Proposition 2.6. Let $n \geq 3$ and $N_{1}, N_{2}, N_{3}$ be positive numbers. Suppose that supp $\widehat{f}$, supp $\widehat{g}$, supp $\widehat{h}$ are contained in $A\left(N_{1}\right), A\left(N_{2}\right), A\left(N_{3}\right)$, respectively. Then for any admissible pair $(\widetilde{q}, \widetilde{r})$,

$$
\|\mathcal{H}(f, g, h)\|_{L_{t}^{\tilde{q}^{\prime}} L_{x}^{\tilde{r}^{\prime}}} \lesssim C\left(N_{1}, N_{2}, N_{3}\right)\|f\|_{L^{2}}\|g\|_{L^{2}}\|h\|_{L^{2}}
$$

where

$$
C\left(N_{1}, N_{2}, N_{3}\right)=\left(\frac{\min \left(N_{1}, N_{2}, N_{3}\right)}{\max \left(N_{1}, N_{2}, N_{3}\right)}\right)^{1 / 2} .
$$

For the proof it is enough to consider two endpoints $\left(\widetilde{q}^{\prime}, \widetilde{r}^{\prime}\right)=(1,2),\left(2, \frac{2 n}{n+2}\right)$ because interpolation gives the remaining estimates. By symmetry we may assume that $N_{1} \geq N_{2}$. On account of scaling structure (Lemma 2.5) we may also assume that

$$
1=\max \left(N_{1}, N_{3}\right) .
$$


Hence we can further assume that $1 \gg \min \left(N_{1}, N_{2}, N_{3}\right)$ since the desired estimates are already contained in Lemma 2.5 when $N_{1} \sim N_{2} \sim N_{3}$. Then we prove Proposition 2.6 by considering the cases $N_{1} \gg N_{2}$ and $N_{1} \sim N_{2}$, separately. To begin with, we recall the following simple lemma which can be easily shown by using the Strichartz estimates and rescaling.

Lemma 2.7. If supp $\widehat{f} \subset A(N)$, for $q, r \geq 2$ satisfying $n / r+2 / q \leq n / 2$

$$
\left\|e^{i t \Delta} f\right\|_{L_{t}^{q} L_{x}^{r}} \lesssim N^{\frac{n}{2}-\frac{n}{r}-\frac{2}{q}}\|f\|_{2} \text {. }
$$

Case $N_{1} \gg N_{2}$. In this case the spatial Fourier support of $e^{i t \Delta} f e^{i t \Delta} g$ is contained in $A\left(2 N_{1}\right)$ because $N_{1} \gg N_{2}$. Hence, $|\nabla|^{2-n} \sim N_{1}^{2-n}$. Using Hörmander-Mikhlin multiplier theorem we see

$$
\|\mathcal{H}(f, g, h)\|_{L_{t}^{\tilde{q}^{\prime}} L_{x}^{\tilde{r}^{\prime}}} \lesssim N_{1}^{2-n}\left\|e^{i t \Delta} f e^{i t \Delta} g e^{i t \Delta} h\right\|_{L_{t}^{\tilde{q}^{\prime}} L_{x}^{\tilde{r}^{\prime}}}
$$

We now have three subcases $(i) 1=N_{3} \geq N_{1} \gg N_{2}$, (ii) $1=N_{1} \geq N_{3} \geq N_{2}$, (iii) $1=N_{1} \gg N_{2} \geq N_{3}$.

We consider the case $(i)$ first. Taking $\left(\widetilde{q}^{\prime}, \widetilde{r}^{\prime}\right)=\left(2, \frac{2 n}{n+2}\right)$ in (2.7) and using Hölder's inequality, it follows that

$$
\|\mathcal{H}(f, g, h)\|_{L_{t}^{2} L_{x}^{\frac{2 n}{n+2}}} \lesssim N_{1}^{2-n}\left\|e^{i t \Delta} g e^{i t \Delta} h\right\|_{L_{t}^{2} L_{x}^{2}}\left\|e^{i t \Delta} f\right\|_{L_{t}^{\infty} L_{x}^{n}}
$$

Since $1 \gg N_{2}$, using (2.2) and Bernstein's inequality (or Lemma 2.7), we get

$$
\|\mathcal{H}(f, g, h)\|_{L_{t}^{2} L_{x}^{\frac{2 n}{n+2}}} \lesssim N_{2}^{\frac{1}{2}}\left(\frac{N_{2}}{N_{1}}\right)^{\frac{n-2}{2}}\|f\|_{L^{2}}\|g\|_{L^{2}}\|h\|_{L^{2}} .
$$

This gives the desired estimate for $\left(\widetilde{q}^{\prime}, \widetilde{r}^{\prime}\right)=\left(2, \frac{2 n}{n+2}\right)$. Similarly, taking $\left(\widetilde{q}^{\prime}, \widetilde{r}^{\prime}\right)=(2,1)$ in (2.7) and using Hölder's inequality, we have

$$
\|\mathcal{H}(f, g, h)\|_{L_{t}^{1} L_{x}^{2}} \lesssim N_{1}^{2-n}\left\|e^{i t \Delta} f\right\|_{L_{t}^{2} L_{x}^{\infty}}\left\|e^{i t \Delta} g e^{i t \Delta} h\right\|_{L_{t}^{2} L_{x}^{2}}
$$

By (2.2) and Lemma 2.7, we get

$$
\|\mathcal{H}(f, g, h)\|_{L_{t}^{1} L_{x}^{2}} \lesssim N_{2}^{\frac{1}{2}}\left(\frac{N_{2}}{N_{1}}\right)^{\frac{n-2}{2}}\|f\|_{L^{2}}\|g\|_{L^{2}}\|h\|_{L^{2}} .
$$

Hence we get the desired bound for $\left(\widetilde{q}^{\prime}, \widetilde{r}^{\prime}\right)=(2,1)$ because $N_{2} \leq N_{1}$.

The remaining two cases $(i i),(i i i)$ can be handled similarly. In fact, for the case (ii), repeating the same argument, using (2.7), (2.2) and Lemma 2.7 we see that

$$
\|\mathcal{H}(f, g, h)\|_{L_{t}^{2} L_{x}^{\frac{2 n}{n+2}}} \lesssim\left\|e^{i t \Delta} f e^{i t \Delta} g\right\|_{L_{t}^{2} L_{x}^{2}}\left\|e^{i t \Delta} h\right\|_{L_{t}^{\infty} L_{x}^{n}} \lesssim N_{2}^{\frac{n-1}{2}} N_{3}^{\frac{n-2}{2}}\|f\|_{L^{2}}\|g\|_{L^{2}}\|h\|_{L^{2}}
$$

and

$$
\|\mathcal{H}(f, g, h)\|_{L_{t}^{1} L_{x}^{2}} \lesssim\left\|e^{i t \Delta} f e^{i t \Delta} g\right\|_{L_{t}^{2} L_{x}^{2}}\left\|e^{i t \Delta} h\right\|_{L_{t}^{2} L_{x}^{\infty}} \lesssim N_{2}^{\frac{n-1}{2}} N_{3}^{\frac{n-2}{2}}\|f\|_{L^{2}}\|g\|_{L^{2}}\|h\|_{L^{2}} .
$$

Because $1 \gg N_{2}$. So we get the desired estimate for $\left(\widetilde{q}^{\prime}, \widetilde{r}^{\prime}\right)=(1,2),\left(2, \frac{2 n}{n+2}\right)$. 
Finally, for case $($ iii $)$, then by (2.7) and repeating the same argument one can show that for $\left(\widetilde{q}^{\prime}, \widetilde{r}^{\prime}\right)=(1,2),\left(2, \frac{2 n}{n+2}\right)$,

$$
\|\mathcal{H}(f, g, h)\|_{L_{t}^{\widetilde{q}^{\prime}} L_{x}^{\tilde{r}^{\prime}}} \lesssim N_{3}^{\frac{n-1}{2}} N_{2}^{\frac{n-2}{2}}\|f\|_{L^{2}}\|g\|_{L^{2}}\|h\|_{L^{2}}
$$

This completes the proof for the case $N_{1} \gg N_{2}$. Now we turn to the remaining case $N_{1} \sim N_{2}$.

Case $N_{1} \sim N_{2}$. In this case $|\nabla|^{2-n}$ can not be handled simply as before. So we need an additional argument to handle this. We begin with decomposing $|\nabla|^{2-n}$ so that

$$
|\nabla|^{2-n}=\sum_{N: \text { dyadic }} N^{2-n} \psi(|\nabla| / N)
$$

with a cut-off $\psi$ supported in $A(1) \dagger$. Here $m(|\nabla|)$ is the multiplier operator defined by $m(|\nabla|) f=\mathcal{F}^{-1}(m(|\cdot|) \widehat{f})$ for a measurable function $m$. Then we have

$$
\mathcal{H}(f, g, h)=\sum_{N: \text { dyadic }} N^{2-n} \psi(|\nabla| / N)\left(e^{i t \Delta} f e^{i t \Delta} g\right) e^{i t \Delta} h .
$$

We first try to obtain estimates for $\psi(|\nabla| / N)\left(e^{i t \Delta} f e^{i t \Delta} g\right) e^{i t \Delta} h$. We claim that for $\left(\widetilde{q}^{\prime}, \widetilde{r}^{\prime}\right)=(1,2),\left(2, \frac{2 n}{n+2}\right)$,

$$
\begin{aligned}
\| \psi(|\nabla| / N) & \left(e^{i t \Delta} f e^{i t \Delta} g\right) e^{i t \Delta} h \|_{L_{t}^{\widetilde{q}^{\prime}} L_{x}^{\widetilde{r}^{\prime}}} \\
& \lesssim N^{\frac{n-2}{2}}\left(\min \left(N, N_{3}\right)\right)^{\frac{n-1}{2}}\|f\|_{L^{2}}\|g\|_{L^{2}}\|h\|_{L^{2}}
\end{aligned}
$$

To show the claim we break $f$ and $g$ into functions having Fourier supports in cubes of side length $2^{-2} N$. Let $\{Q\}$ be a collection of (essentially) disjoint cubes of side length $2^{-2} N$ covering $A\left(N_{1}\right)$ and we set

$$
\widehat{f}_{Q}=\chi_{Q}(\xi) \widehat{f}, \quad \widehat{g}_{Q}=\chi_{Q}(\xi) \widehat{g}
$$

Then we have $f=\sum_{Q} f_{Q}$ and $g=\sum_{Q} g_{Q}$, and we may assume that $Q \subset A\left(N_{1}\right)$ because $N_{1} \sim N_{2}$. Then it follows that

$$
\begin{aligned}
\text { LHS of }(\underline{2.9)}) & \lesssim \sum_{Q, Q^{\prime}}\left\|\psi(|\nabla| / N)\left(e^{i t \Delta} f_{Q} e^{i t \Delta} g_{Q^{\prime}}\right) e^{i t \Delta} h\right\|_{L_{t}^{\tilde{q}^{\prime}} L_{x}^{\tilde{r}^{\prime}}} \\
& \lesssim \sum_{\operatorname{dist}\left(Q,-Q^{\prime}\right) \leq 4 N}\left\|\psi(|\nabla| / N)\left(e^{i t \Delta} f_{Q} e^{i t \Delta} g_{Q^{\prime}}\right) e^{i t \Delta} h\right\|_{L_{t}^{\tilde{q}^{\prime}} L_{x}^{\tilde{r}^{\prime}}}
\end{aligned}
$$

${ }^{\dagger}$ Actually the sum is taken over $N \lesssim \max \left(N_{1}, N_{2}\right)$ because of the supports of $\widehat{f}, \widehat{g}$. 
because $\psi(|\nabla| / N)\left(e^{i t \Delta} f_{Q} e^{i t \Delta} g_{Q^{\prime}}\right)=0$ if dist $\left(Q,-Q^{\prime}\right)>4 N$. Hence it is enough to show that for $\left(\widetilde{q}^{\prime}, \widetilde{r}^{\prime}\right)=(1,2),\left(2, \frac{2 n}{n+2}\right)$,

$$
\begin{aligned}
& \left\|\psi(|\nabla| / N)\left(e^{i t \Delta} f_{Q} e^{i t \Delta} g_{Q^{\prime}}\right) e^{i t \Delta} h\right\|_{L_{t}^{\widetilde{q}^{\prime}} L_{x}^{\tilde{r}^{\prime}}} \\
& \quad \lesssim N^{\frac{n-2}{2}}\left(\min \left(N, N_{3}\right)\right)^{\frac{n-1}{2}}\left\|f_{Q}\right\|_{L^{2}}\left\|g_{Q^{\prime}}\right\|_{L^{2}}\|h\|_{L^{2}}
\end{aligned}
$$

Indeed, from (2.10) and (2.11) we get

$$
\begin{aligned}
\text { LHS of (2.9) }) & \lesssim N^{\frac{n-2}{2}}\left(\min \left(N, N_{3}\right)\right)^{\frac{n-1}{2}} \sum_{\operatorname{dist}\left(Q,-Q^{\prime}\right) \leq 4 N}\left\|f_{Q}\right\|_{L^{2}}\left\|g_{Q^{\prime}}\right\|_{L^{2}}\|h\|_{L^{2}} \\
& \lesssim N^{\frac{n-2}{2}}\left(\min \left(N, N_{3}\right)\right)^{\frac{n-1}{2}}\left(\sum_{Q}\left\|f_{Q}\right\|_{L^{2}}^{2}\right)^{\frac{1}{2}}\left(\sum_{Q^{\prime}}\left\|g_{Q^{\prime}}\right\|_{L^{2}}^{2}\right)^{\frac{1}{2}}\|h\|_{L^{2}} \\
& \lesssim N^{\frac{n-2}{2}}\left(\min \left(N, N_{3}\right)\right)^{\frac{n-1}{2}}\|f\|_{L^{2}}\|g\|_{L^{2}}\|h\|_{L^{2}} .
\end{aligned}
$$

For the second and third inequalities we used Cauchy-Schwarz inequality and orthogonality, respectively. Hence matters are reduced to showing (2.11).

Now observe that

$$
\begin{aligned}
\psi(|\nabla| / N)\left(e^{i t \Delta} f_{Q} e^{i t \Delta} g_{Q^{\prime}}\right) & =\iint e^{i x \cdot(\xi+\eta)-i t\left(|\xi|^{2}+|\eta|^{2}\right)} \\
& \times \phi\left(\xi / N-\xi_{0}\right) \psi((\xi+\eta) / N) \phi\left(\eta / N-\eta_{0}\right) \widehat{f}_{Q}(\xi) \widehat{g}_{Q^{\prime}}(\eta) d \xi d \eta
\end{aligned}
$$

for some $\xi_{0}, \eta_{0} \in \mathbb{R}^{n}$ and $\phi$ supported in $B(0,1)$. Expanding $\Psi(\xi, \eta)=\phi(\xi-$ $\left.\xi_{0}\right) \psi(\xi+\eta) \phi\left(\eta-\eta_{0}\right)$ into Fourier series on the cube of side length $2 \pi$ which contains the support of $\Psi$, we have

$$
\phi\left(\xi-\xi_{0}\right) \psi(\xi+\eta) \phi\left(\eta-\eta_{0}\right)=\sum_{k, l \in \mathbb{Z}^{n}} C_{k, l} e^{i(k \cdot \xi+l \cdot \eta)}
$$

with $\sum_{k, l}\left|C_{k, l}\right| \leq C$, independent of $\xi_{0}, \eta_{0}$. Plugging this in the above we get

$$
\psi(|\nabla| / N)\left(e^{i t \Delta} f_{Q} e^{i t \Delta} g_{Q^{\prime}}\right)=\sum_{k, l \in \mathbb{Z}^{n}} C_{k, l} e^{i t \Delta} f_{Q}^{k} e^{i t \Delta} g_{Q^{\prime}}^{l}
$$

with $\left\|f_{Q}^{k}\right\|_{L^{2}}=\left\|f_{Q}\right\|_{L^{2}}$ and $\left\|g_{Q^{\prime}}^{l}\right\|_{L^{2}}=\left\|g_{Q^{\prime}}\right\|_{L^{2}}$ for all $k, l$. Hence to show (2.11) it suffices to show that for $\left(\widetilde{q}^{\prime}, \widetilde{r}^{\prime}\right)=(1,2),\left(2, \frac{2 n}{n+2}\right)$,

$$
\left\|e^{i t \Delta} f e^{i t \Delta} g e^{i t \Delta} h\right\|_{L_{t}^{\tilde{q}^{\prime}} L_{x}^{\tilde{r}^{\prime}}} \lesssim N^{\frac{2-n}{2}}\left(\min \left(N, N_{3}\right)\right)^{\frac{n-1}{2}}\|f\|_{L^{2}}\|g\|_{L^{2}}\|h\|_{L^{2}}
$$

whenever $\widehat{f}, \widehat{g}$ are supported in cubes $Q, Q^{\prime} \subset A\left(N_{1}\right)$ of side length $N$ and $\widehat{h}$ is supported in $A\left(N_{3}\right)$. Note that dist $(\operatorname{supp} \widehat{f}$, supp $\widehat{h}) \sim 1$ and $\operatorname{dist}(\operatorname{supp} \widehat{g}$, supp $\widehat{h}) \sim 1$ because there are only two possible cases $1=N_{1} \sim N_{2} \gg N_{3}, 1=N_{3} \gg N_{1} \sim N_{2}$. 
Hence, by Hölder's inequality, (2.2) and Lemma 2.7, we get

$$
\begin{aligned}
\left\|e^{i t \Delta} f e^{i t \Delta} g e^{i t \Delta} h\right\|_{L_{t}^{2} L_{x}^{\frac{2 n}{n+2}}} & \lesssim\left\|e^{i t \Delta} f\right\|_{L_{t}^{\infty} L_{x}^{n}}\left\|e^{i t \Delta} g e^{i t \Delta} h\right\|_{L_{t}^{2} L_{x}^{2}} \\
& \lesssim N^{\frac{n-2}{2}}\left(\min \left(N, N_{3}\right)\right)^{\frac{n-1}{2}}\|f\|_{L^{2}}\|g\|_{L^{2}}\|h\|_{L^{2}},
\end{aligned}
$$

and

$$
\begin{aligned}
\|\mathcal{H}(f, g, h)\|_{L_{t}^{1} L_{x}^{2}} & \lesssim\left\|e^{i t \Delta} f e^{i t \Delta} g\right\|_{L_{t}^{2} L_{x}^{2}}\left\|e^{i t \Delta} h\right\|_{L_{t}^{2} L_{x}^{\infty}} \\
& \lesssim N^{\frac{n-2}{2}}\left(\min \left(N, N_{3}\right)\right)^{\frac{n-1}{2}}\|f\|_{L^{2}}\|g\|_{L^{2}}\|h\|_{L^{2}}
\end{aligned}
$$

Hence we get (2.9).

We now consider two cases $1=N_{1} \sim N_{2} \gg N_{3}, 1=N_{3} \gg N_{1} \sim N_{2}$, separately. When $1=N_{1} \sim N_{2} \gg N_{3}$, from (2.8), triangle inequality and (2.9) we get

$$
\begin{aligned}
\|\mathcal{H}(f, g, h)\|_{L_{t}^{\tilde{q}^{\prime}} L_{x}^{\widetilde{r}^{\prime}}} & \lesssim \sum_{N \leq 4} N^{2-n}\left\|\psi(|\nabla| / N)\left(e^{i t \Delta} f e^{i t \Delta} g\right) e^{i t \Delta} h\right\|_{L_{t}^{\tilde{q}^{\prime}} L_{x}^{\widetilde{r}^{\prime}}} \\
& \lesssim \sum_{N \leq 4} N^{\frac{2-n}{2}}\left(\min \left(N, N_{3}\right)\right)^{\frac{n-1}{2}}\|f\|_{L^{2}}\|g\|_{L^{2}}\|h\|_{L^{2}}
\end{aligned}
$$

Summation in $N$ gives

$$
\|\mathcal{H}(f, g, h)\|_{L_{t}^{\widetilde{q}^{\prime}} L_{x}^{\widetilde{r}^{\prime}}} \lesssim N_{3}^{\frac{1}{2}}\|f\|_{L^{2}}\|g\|_{L^{2}}\|h\|_{L^{2}}
$$

This proves the case $(i) 1=N_{1} \sim N_{2} \gg N_{3}$. When $1=N_{3} \gg N_{1} \sim N_{2}$ note that the summation is taken over $N \lesssim N_{1}$. By (2.8), triangle inequality and (2.9) we get

$$
\|\mathcal{H}(f, g, h)\|_{L_{t}^{\widetilde{q}^{\prime}} L_{x}^{\widetilde{r}^{\prime}}} \lesssim \sum_{N \lesssim N_{1}} N^{\frac{1}{2}}\|f\|_{L^{2}}\|g\|_{L^{2}}\|h\|_{L^{2}}
$$

The desired estimate follow from summation in $N$. This completes the proof of Proposition 2.6.

Before closing this subsection we state a slightly strengthened version of Proposition 2.6 which is to be used in Section 4.

Corollary 2.8. Let $i=1,2,3$. If $N_{i} \sim \min \left(N_{1}, N_{2}, N_{3}\right)$, then Proposition [2.6 remains valid with $A\left(N_{i}\right)$ replaced by $B\left(0, N_{i}\right)$ in the assumption.

Proof. When all of $N_{1}, N_{2}, N_{3} \sim \min \left(N_{1}, N_{2}, N_{3}\right)$, (2.6) trivially holds by Lemma 2.5. If only one $N_{i}$ of $N_{1}, N_{2}, N_{3} \sim \min \left(N_{1}, N_{2}, N_{3}\right)$, then by decomposition of $B\left(0, N_{i}\right)$ into dyadic shells, applying Proposition 2.6 to each dyadic shell and direct summation of geometric series one can easily see that (2.6) holds. The other possibility is that two of $N_{1}, N_{2}, N_{3} \sim \min \left(N_{1}, N_{2}, N_{3}\right)$. In this case we only need to consider two cases $N_{2} \sim N_{3} \ll N_{1}$ and $N_{1} \sim N_{2} \ll N_{3}$ by symmetry between $N_{1}$ and $N_{2}$. For both cases one can see without difficulty that the argument for the 
proof of Proposition 2.6 works for either supp $\widehat{g} \subset B\left(0, N_{2}\right)$ and supp $\widehat{h} \subset B\left(0, N_{3}\right)$ or supp $\widehat{f} \subset B\left(0, N_{1}\right)$ and supp $\widehat{g} \subset B\left(0, N_{2}\right)$.

We now prove Theorem 1.2 by showing (1.2), (1.3), separately.

2.3. Proof of (1.2) . For simplicity we denote by $f_{N_{j}}(j=1,2,3)$ the LittlewoodPaley projection $P_{N_{j}} f$ of $f$. Then we decompose

$$
\begin{aligned}
\mathcal{H}(f, g, \nabla h) & =\sum_{N_{1}, N_{2}, N_{3}} \mathcal{H}\left(f_{N_{1}}, g_{N_{2}}, \nabla h_{N_{3}}\right) \\
& =\sum_{N_{1} \leq N_{2}} \sum_{N_{3}} \mathcal{H}\left(f_{N_{1}}, g_{N_{2}}, \nabla h_{N_{3}}\right)+\sum_{N_{1}>N_{2}} \sum_{N_{3}} \mathcal{H}\left(f_{N_{1}}, g_{N_{2}}, \nabla h_{N_{3}}\right) .
\end{aligned}
$$

By symmetry it is enough to handle the first one because the second can be handled similarly. Then we have three possible cases; $N_{3} \leq N_{1} \leq N_{2}, N_{1} \leq N_{3} \leq N_{2}$ and $N_{1} \leq N_{2} \leq N_{3}$. We separately treat the summation of each case.

Case $N_{3} \leq N_{1} \leq N_{2}$. This case is the easiest. It can be handled by using the Strichartz estimates only. We claim that for any positive $s_{1}, s_{2}, s_{3}$ with $\sum s_{i}=1$,

$$
\left\|\sum_{N_{3} \leq N_{1} \leq N_{2}} \mathcal{H}\left(f_{N_{1}}, g_{N_{2}}, \nabla h_{N_{3}}\right)\right\|_{L_{t}^{\tilde{q}^{\prime}} L_{x}^{\tilde{r}^{\prime}}} \lesssim\|f\|_{\dot{H}^{s_{1}}}\|g\|_{\dot{H}^{s_{2}}}\|h\|_{\dot{H}^{s_{3}}} .
$$

By setting $N_{2}=N_{3} N_{4} \equiv N_{34}$ we write

$$
\sum_{N_{3} \leq N_{1} \leq N_{2}} \mathcal{H}\left(f_{N_{1}}, g_{N_{2}}, \nabla h_{N_{3}}\right)=\sum_{N_{4} \geq 1} \sum_{N_{3}=-\infty}^{\infty} \sum_{N_{3} \leq N_{1} \leq N_{34}} \mathcal{H}\left(f_{N_{1}}, g_{N_{34}}, \nabla h_{N_{3}}\right) .
$$

Using Lemma 2.5 we get

$$
\left\|\mathcal{H}\left(f_{N_{1}}, g_{N_{34}}, \nabla h_{N_{3}}\right)\right\|_{L_{t}^{\tilde{q}^{\prime}} L_{x}^{\tilde{r}^{\prime}}} \lesssim N_{3}\left\|f_{N_{1}}\right\|_{L^{2}}\left\|g_{34}\right\|_{L^{2}}\left\|h_{3}\right\|_{L^{2}}
$$

So, the norm $\left\|\sum_{N_{3} \leq N_{1} \leq N_{2}} \mathcal{H}\left(f_{N_{1}}, g_{N_{2}}, \nabla h_{N_{3}}\right)\right\|_{L_{t}^{\tilde{q}^{\prime} L_{x}^{\tilde{r}^{\prime}}}}$ is bounded by

$$
C \sum_{N_{4} \geq 1} \sum_{N_{3}} \sum_{N_{3} \leq N_{1} \leq N_{34}} N_{3} N_{1}^{-s_{1}} N_{34}^{-s_{2}} N_{3}^{-s_{3}}\left(N_{1}^{s_{1}}\left\|f_{N_{1}}\right\|_{L^{2}}\right)\left(N_{34}^{s_{2}}\left\|g_{N_{34}}\right\|_{L^{2}}\right)\left(N_{3}^{s_{3}}\left\|h_{N_{3}}\right\|_{L^{2}}\right) \text {. }
$$

It is also bounded again by

$$
C\|f\|_{\dot{H}^{s_{1}}} \sum_{N_{4} \geq 1} N_{4}^{-s_{2}} \sum_{N_{3}}\left(N_{34}^{s_{2}}\left\|g_{N_{34}}\right\|_{L^{2}}\right)\left(N_{3}^{s_{3}}\left\|h_{N_{3}}\right\|_{L^{2}}\right) .
$$

Then Cauchy-Schwarz inequality yields the desired bound.

Case $N_{1} \leq N_{3} \leq N_{2}$. In this case we set $N_{2}=N_{1} N_{4} \equiv N_{14}$ and write

$$
\sum_{N_{1} \leq N_{3} \leq N_{2}} \mathcal{H}\left(f_{N_{1}}, g_{N_{2}}, \nabla h_{N_{3}}\right)=\sum_{N_{4} \geq 1} \sum_{N_{1}} \sum_{N_{1} \leq N_{3} \leq N_{14}} \mathcal{H}\left(f_{N_{1}}, g_{N_{14}}, \nabla h_{N_{3}}\right) .
$$

Using triangle inequality and Proposition 2.6 we see

$$
\left\|\mathcal{H}\left(f_{N_{1}}, g_{N_{14}}, \nabla h_{N_{3}}\right)\right\|_{L_{t}^{\tilde{q}^{\prime}} L_{x}^{\tilde{r}^{\prime}}} \lesssim N_{3} N_{4}^{-\frac{1}{2}}\left\|f_{1}\right\|_{L^{2}}\left\|g_{14}\right\|_{L^{2}}\left\|h_{3}\right\|_{L^{2}}
$$


Hence the norm $\left\|\sum_{N_{1} \leq N_{3} \leq N_{2}} \mathcal{H}\left(f_{N_{1}}, g_{N_{2}}, \nabla h_{N_{3}}\right)\right\|_{L_{t}^{\tilde{q}^{\prime}} L_{x}^{\tilde{r}^{\prime}}}$ is bounded by

$$
\|h\|_{\dot{H}^{s_{3}}} \sum_{N_{4} \geq 1} N_{4}^{-\frac{1}{2}} \sum_{N_{1}} \sum_{N_{1} \leq N_{3} \leq N_{14}} N_{3}^{1-s_{3}} N_{1}^{-s_{1}} N_{14}^{-s_{2}}\left(N_{1}^{s_{1}}\left\|f_{N_{1}}\right\|_{L^{2}}\right)\left(N_{14}^{s_{2}}\left\|g_{N_{14}}\right\|_{L^{2}}\right) \text {. }
$$

Taking summation in $N_{3}$ and using Schwarz's inequality in $N_{1}$, we bounds this by

$$
C\|f\|_{\dot{H}^{s_{1}}}\|g\|_{\dot{H}^{s_{2}}}\|h\|_{\dot{H}^{s_{3}}} \sum_{N_{4} \geq 1} N_{4}^{s_{1}-\frac{1}{2}}
$$

Note that $s_{1}<\frac{1}{2}$ because $s_{3}>\frac{1}{2}$. Hence we get the desired.

Case $N_{1} \leq N_{2} \leq N_{3}$. We set $N_{3}=N_{1} N_{4} \equiv N_{14}$ and write

$$
\sum_{N_{1} \leq N_{2} \leq N_{3}} \mathcal{H}\left(f_{N_{1}}, g_{N_{2}}, \nabla h_{N_{3}}\right)=\sum_{N_{4} \geq 1} \sum_{N_{1}} \sum_{N_{1} \leq N_{2} \leq N_{14}} \mathcal{H}\left(f_{N_{1}}, g_{N_{2}}, \nabla h_{N_{14}}\right) .
$$

Using triangle inequality and Proposition 2.6 we have

$$
\begin{aligned}
\left\|\sum_{N_{1} \leq N_{2} \leq N_{3}} \mathcal{H}\left(f_{N_{1}}, g_{N_{2}}, \nabla h_{N_{3}}\right)\right\|_{L_{t}^{\tilde{q}^{\prime}} L_{x}^{\tilde{r}^{\prime}}} \\
\qquad \sum_{N_{4} \geq 1} \sum_{N_{1}} \sum_{N_{1} \leq N_{2} \leq N_{14}} N_{14} N_{4}^{-\frac{1}{2}}\left\|f_{1}\right\|_{L^{2}}\left\|g_{2}\right\|_{L^{2}}\left\|h_{14}\right\|_{L^{2}}
\end{aligned}
$$

The left hand side of the above is bounded by

$$
C\|g\|_{\dot{H}^{s_{2}}} \sum_{N_{4} \geq 1} N_{4}^{-\frac{1}{2}} \sum_{N_{1}} \sum_{N_{1} \leq N_{2} \leq N_{14}} N_{1}^{-s_{1}} N_{2}^{-s_{2}} N_{14}^{1-s_{3}}\left(N_{1}^{s_{1}}\left\|f_{N_{1}}\right\|_{L^{2}}\right)\left(N_{14}^{s_{3}}\left\|h_{N_{14}}\right\|_{L^{2}}\right) .
$$

Taking summation in $N_{2}$ and using Schwarz's inequality in $N_{1}$, the above is bounded by a constant multiple of $\|f\|_{\dot{H}^{s_{1}}}\|g\|_{\dot{H}^{s_{2}}}\|h\|_{\dot{H}^{s_{3}}} \sum_{N_{4} \geq 1} N_{4}^{-s_{3}+\frac{1}{2}}$. Since $s_{3}>\frac{1}{2}$, we get the desired.

\subsection{Proof of (1.3) $)$ We decompose}

$$
\mathcal{H}(\nabla f, g, h)=\sum_{N_{1}, N_{2}, N_{3}} \mathcal{H}\left(\nabla f_{N_{1}}, g_{N_{2}}, h_{\left.N_{3}\right)}\right.
$$

There is no obvious symmetry. We should consider the following six cases:

$$
\begin{aligned}
& \text { (i) } N_{1} \geq N_{2} \geq N_{3}, \quad \text { (ii) } N_{1} \geq N_{3} \geq N_{2}, \text { (iii) } N_{3} \geq N_{1} \geq N_{2}, \\
& \text { (iv) } N_{2} \geq N_{1} \geq N_{3}, \text { (v) } N_{2} \geq N_{3} \geq N_{1}, \text { (vi) } N_{3} \geq N_{2} \geq N_{1}
\end{aligned}
$$

As expected, the cases $(i)$ and $(i i)$ are the major parts. The others are sort of minor terms. Each of the cases can be handled by the same argument as before. 
Case $(i) N_{1} \geq N_{2} \geq N_{3}$ : To begin with, we set $N_{1}=N_{34}$. By the triangle inequality and rearrangement of the summation we get

$$
\begin{array}{rl}
\| \sum_{N_{3} \leq N_{2} \leq N_{1}} & \mathcal{H}\left(\nabla f_{N_{1}}, g_{N_{2}}, h_{\left.N_{3}\right)} \|_{L_{t}^{\tilde{q}^{\prime}} L_{x}^{\tilde{r}^{\prime}}}\right. \\
& \lesssim \sum_{N_{4} \geq 1} \sum_{N_{1}} \sum_{N_{34} \geq N_{2} \geq N_{3}} \| \mathcal{H}\left(\nabla f_{N_{34}}, g_{N_{2}}, h_{\left.N_{3}\right)} \|_{L_{t}^{\tilde{q}^{\prime}} L_{x}^{\tilde{r}^{\prime}}} \cdot\right.
\end{array}
$$

Applying Proposition 2.6 we bound the left hand side of the above by

$$
\|g\|_{H^{s_{2}}} \sum_{N_{4} \geq 1} N_{4}^{-\frac{1}{2}} \sum_{N_{3}} \sum_{N_{34} \geq N_{2} \geq N_{3}} N_{34}^{1-s_{1}} N_{2}^{-s_{2}} N_{3}^{-s_{3}}\left(N_{34}^{s_{1}}\left\|f_{N_{34}}\right\|_{L^{2}}\right)\left(N_{3}^{s 3}\left\|h_{N_{3}}\right\|_{L^{2}}\right) .
$$

Taking summ in $N_{2}$ and using Schwarz's inequality in $N_{1}$, we see that

$$
\left\|\sum_{N_{3} \leq N_{2} \leq N_{1}} \mathcal{H}\left(\nabla f_{N_{1}}, g_{N_{2}}, h_{N_{3}}\right)\right\|_{L_{t}^{\tilde{q}^{\prime}} L_{x}^{\tilde{r}^{\prime}}} \lesssim\|f\|_{\dot{H}^{s_{1}}}\|g\|_{\dot{H}^{s_{2}}}\|h\|_{\dot{H}^{s_{3}}} \sum_{N_{4} \geq 1} N_{4}^{-s_{1}+\frac{1}{2}} .
$$

Since $s_{1}>\frac{1}{2}$, we get the desired.

Case ( $i i) N_{1} \geq N_{3} \geq N_{2}$ : We set $N_{1}=N_{24}$ and rearrange the summation such that

$$
\sum_{N_{1} \geq N_{3} \geq N_{2}}=\sum_{N_{1}} \sum_{N_{2}} \sum_{N_{24} \geq N_{3} \geq N_{2}} .
$$

While applying triangle inequality and Proposition 2.6, the only difference to the previous case $(i)$ is that $N_{3}$ is replaced by $N_{2}$. Hence by the same argument we get

$$
\left\|\sum_{N_{1} \geq N_{3} \geq N_{2}}(\cdot)\right\|_{L_{t}^{\tilde{q}^{\prime}} L_{x}^{\tilde{r}^{\prime}}} \lesssim\|f\|_{\dot{H}^{s_{1}}}\|g\|_{\dot{H}^{s_{2}}}\|h\|_{\dot{H}^{s_{3}}} \sum_{N_{4} \geq 1} N_{4}^{-s_{1}+\frac{1}{2}} .
$$

Since $s_{1}>\frac{1}{2}$, we get the desired.

The remaining cases $(i i i)-(v i)$ can be handled by the same way. Repeating the argument one can show

$$
\begin{aligned}
\left\|\sum_{N_{3} \geq N_{1} \geq N_{2}}(\cdot)\right\|_{L_{t}^{\tilde{q}^{\prime}} L_{x}^{\tilde{r}^{\prime}}}, \lesssim\|f\|_{\dot{H}^{s_{1}}}\|g\|_{\dot{H}^{s_{2}}}\|h\|_{\dot{H}^{s_{3}}} \sum_{N_{4} \geq 1} N_{4}^{-\frac{1}{2}+s_{2}}, \\
\left\|\sum_{N_{2} \geq N_{1} \geq N_{3}}(\cdot)\right\|_{L_{t}^{\tilde{q}^{\prime}} L_{x}^{\tilde{r}^{\prime}}} \lesssim\|f\|_{\dot{H}^{s_{1}}}\|g\|_{\dot{H}^{s_{2}}}\|h\|_{\dot{H}^{s_{3}}} \sum_{N_{4} \geq 1} N_{4}^{-\frac{1}{2}+s_{3}} .
\end{aligned}
$$

Since $s_{1}>\frac{1}{2}, s_{2}, s_{3}<\frac{1}{2}$, we get the desired. One can also show

$$
\begin{aligned}
\left\|\sum_{N_{3} \geq N_{2} \geq N_{1}}(\cdot)\right\|_{L_{t}^{\tilde{q}^{\prime}} L_{x}^{\tilde{r}^{\prime}}}, \lesssim\|f\|_{\dot{H}^{s_{1}}}\|g\|_{\dot{H}^{s_{2}}}\|h\|_{\dot{H}^{s_{3}}} \sum_{N_{4} \geq 1} N_{4}^{-\frac{1}{2}-s_{3}}, \\
\left\|\sum_{N_{2} \geq N_{3} \geq N_{1}}(\cdot)\right\|_{L_{t}^{\tilde{q}^{\prime}} L_{x}^{\tilde{r}^{\prime}}} \lesssim\|f\|_{\dot{H}^{s_{1}}}\|g\|_{\dot{H}^{s_{2}}}\|h\|_{\dot{H}^{s_{3}}} \sum_{N_{4} \geq 1} N_{4}^{-\frac{1}{2}-s_{2}} .
\end{aligned}
$$

Therefore this completes the proof of Theorem 1.2 . 


\section{Smoothing properties}

In this section we prove Theorem 1.3, The proof relies on the arguments using the Bourgain space $\mathbf{X}^{s, b}$ for $s, b \in \mathbb{R}$. It consists of the functions $u$ such that

$$
\|u\|_{\mathbf{X}^{s, b}} \equiv\left(\iint\langle\xi\rangle^{2 s}\left\langle\tau-|\xi|^{2}\right\rangle^{2 b}|\widetilde{u}(\tau, \xi)|^{2} d \tau d \xi\right)^{\frac{1}{2}}<\infty
$$

where $\widetilde{u}(\tau, \xi)$ is the time-space Fourier transform of $u$. We also use the norm $\mathbf{X}^{s, b}\left(J_{T}\right)$ for time interval $J_{T}=[0, T]$ defined as

$$
\|u\|_{\mathbf{X}^{s, b}\left(J_{T}\right)} \equiv \inf \left\{\|\varphi\|_{\mathbf{X}^{s, b}}:\left.\varphi\right|_{J_{T}}=u\right\} .
$$

3.1. Hartree type nonlinearity. In this section $V(u)$ denotes $\kappa|x|^{-2} *|u|^{2}$. Let us invoke $V(u)=c|\nabla|^{2-n}\left(|u|^{2}\right)$ for some constant $c$. Using the $\mathbf{X}^{s, b}$ spaces and Theorem [1.2, one can derive the following.

Proposition 3.1. Let $n \geq 3$. Then for any $s, b>\frac{1}{2}$ there exists $0<\epsilon \ll 1$ such that

$$
\|[V(u) u]\|_{\mathbf{X}^{1,-\frac{1}{2}+\epsilon}} \lesssim\|u\|_{\mathbf{X}^{s, b}}^{3} .
$$

Proof. We first show that for $s_{0}>2+\frac{n}{2}$

$$
\|\mathcal{H}(\nabla f, g, h)\|_{L_{t}^{\infty} L_{x}^{\infty}}+\|\mathcal{H}(f, g, \nabla h)\|_{L_{t}^{\infty} L_{x}^{\infty}} \lesssim\|f\|_{H^{s_{0}}}\|g\|_{H^{s_{0}}}\|h\|_{H^{s_{0}}} .
$$

Here we do not intend to obtain sharp $s_{0}$ but we here are content with some crude estimate which is enough for our purpose. From the Sobolev embedding we note that $\left\|e^{i t \Delta}\langle\nabla\rangle f\right\|_{L_{t}^{\infty} L_{x}^{\infty}} \lesssim\|f\|_{H^{s_{0}}}$. Hence it is enough to show that

$$
\left\||\nabla|^{2-n}\left(e^{i t \Delta}\langle\nabla\rangle f e^{i t \Delta} g\right)\right\|_{L_{t}^{\infty} L_{x}^{\infty}} \lesssim\|f\|_{H^{s_{0}}}\|g\|_{H^{s_{0}}} .
$$

But this follows easily from the observation that

$$
\left\||\nabla|^{2-n} G\right\|_{L_{x}^{\infty}} \lesssim\left(\int_{|\xi| \leq 1}+\int_{|\xi| \geq 1}\right)|\xi|^{2-n}|\widehat{G}(\xi)| d \xi \lesssim\|G\|_{L^{1}}+\|G\|_{H^{1}}
$$

together with Leibniz rule and Schwarz's inequality.

Now interpolating (3.2) with the estimates (1.2) of Theorem 1.2, we see that if $(\tilde{q}, \tilde{r})$ is any admissible pair, $s_{3}>\frac{1}{2}$ and $\sum_{i=1}^{3} s_{i}>1$, then there exist $0<\epsilon_{1}, \epsilon_{2} \ll 1$ such that

$$
\|\mathcal{H}(f, g, \nabla h)\|_{L_{t}^{\tilde{q}^{\prime}+\epsilon_{1}} L_{x}^{\tilde{r}^{\prime}+\epsilon_{2}}} \lesssim\|f\|_{H^{s_{1}}}\|g\|_{H^{s_{2}}}\|h\|_{H^{s_{3}}} .
$$

Similarly, using (3.2) and (1.3), for any admissible pair $(\tilde{q}, \tilde{r})$ and the exponents $s_{1}, s_{2}, s_{3}$ with $s_{1}>\frac{1}{2}, \sum_{i=1}^{3} s_{i}>1$, we can find $\epsilon_{1}$ and $\epsilon_{2}$ such that

$$
\|\mathcal{H}(\nabla f, g, h)\|_{L_{t}^{\tilde{q}^{\prime}+\epsilon_{1}} L_{x}^{\tilde{r}^{\prime}+\epsilon_{2}}} \lesssim\|f\|_{H^{s_{1}}}\|g\|_{H^{s_{2}}}\|h\|_{H^{s_{3}}} .
$$


One may write $u(t, x)=c_{n} \int e^{i t \tau} \int_{\mathbb{R}^{n}} e^{i\left(x \cdot \xi-t|\xi|^{2}\right)} \widetilde{u}\left(\tau-|\xi|^{2}, \xi\right) d \xi d \tau$ by inversion and translation in frequency variables. Hence, we get

$$
\left(|\nabla|^{2-n}(u v) \nabla w\right)(x, t)=\iiint \mathcal{H}\left(f_{\tau}, g_{\tau^{\prime}}, \nabla h_{\tau^{\prime \prime}}\right)(x) d \tau d \tau^{\prime} d \tau^{\prime \prime},
$$

where $\widehat{f}_{\tau}(\xi)=\widetilde{u}\left(\tau-|\xi|^{2}, \xi\right), \widehat{g}_{\tau}(\xi)=\widetilde{v}\left(\tau-|\xi|^{2}, \xi\right), h_{\tau}(\xi)=\widetilde{w}\left(\tau-|\xi|^{2}, \xi\right)$. From (3.3) and Minkowski's inequality it follows that

$$
\left\||\nabla|^{2-n}(u v) \nabla w\right\|_{L_{t}^{\tilde{q}^{\prime}+\epsilon_{1}} L_{x}^{\tilde{r}^{\prime}+\epsilon_{2}}} \lesssim \iiint\left\|f_{\tau}\right\|_{H^{s_{1}}}\left\|g_{\tau^{\prime}}\right\|_{H^{s_{2}}}\left\|h_{\tau^{\prime \prime}}\right\|_{H^{s_{3}}} d \tau d \tau^{\prime} d \tau^{\prime \prime} .
$$

Plancherel's theorem and Schwarz's inequality yield

$$
\left\||\nabla|^{2-n}(u v) \nabla w\right\|_{L_{t}^{\tilde{q}^{\prime}+\epsilon_{1}} L_{x}^{\tilde{r}^{\prime}+\epsilon_{2}}} \lesssim\|u\|_{\mathbf{X}^{s_{1}, b}}\|v\|_{\mathbf{X}^{s_{2}, b}}\|w\|_{\mathbf{X}^{s_{3}, b}}
$$

for any $b>\frac{1}{2}$ and for any $(\tilde{q}, \tilde{r}), s_{1}, s_{2}, s_{3}$ as in (3.3). By repeating the same argument with (3.4), we also get

$$
\left\||\nabla|^{2-n}(\nabla u v) w\right\|_{L_{t}^{\tilde{q}^{\prime}+\epsilon_{1}} L_{x}^{\tilde{r}^{\prime}+\epsilon_{2}}} \lesssim\|u\|_{\mathbf{X}^{s_{1}, b}}\|v\|_{\mathbf{X}^{s_{2}, b}}\|w\|_{\mathbf{X}^{s_{3}, b}}
$$

for any $b>\frac{1}{2}$ and for any $(\tilde{q}, \tilde{r}), s_{1}, s_{2}, s_{3}$ as in (3.3).

We now fix $s, b>1 / 2$. To show (3.1), we need to show that

$$
\|V(u) u\|_{\mathbf{X}^{0,-\frac{1}{2}+\epsilon}},\|\nabla[V(u) u]\|_{\mathbf{X}^{0,-\frac{1}{2}+\epsilon}} \lesssim\|u\|_{\mathbf{X}^{s, b}}^{3}
$$

By duality it suffices to show that

$$
|\langle\psi, U\rangle| \lesssim\|\psi\|_{\mathbf{X}^{0, \frac{1}{2}-\epsilon}}\|u\|_{\mathbf{X}^{s, b}}^{3}
$$

for $U=|\nabla|^{2-n}\left(|u|^{2}\right) u,|\nabla|^{2-n}(\nabla u \bar{u}) u,|\nabla|^{2-n}(u \nabla \bar{u}) u$ and $|\nabla|^{2-n}\left(|u|^{2}\right) \nabla u$. We first handle the case $U=|\nabla|^{2-n}(\nabla u \bar{u}) u$. By Hölder's inequality we have

$$
\left|\left\langle\psi,|\nabla|^{2-n}(\nabla u \bar{u}) u\right\rangle\right| \lesssim\|\psi\|_{L_{t}^{\tilde{q}} L_{x}^{\tilde{r}}}\left\||\nabla|^{2-n}(\nabla u \bar{u}) u\right\|_{L_{t}^{\tilde{q}^{\prime}} L_{x}^{\tilde{r}^{\prime}}}
$$

By (3.6) we can choose $(1 / \tilde{q}, 1 / \tilde{r})$ close enough to the Strichartz line $2 / q+n / r=n / 2$ so that $2 / \tilde{q}+n / \tilde{r}>n / 2$ and $\left\||\nabla|^{2-n}(\nabla u \bar{u}) u\right\|_{L_{t}^{\tilde{q}^{\prime}} L_{x}^{\tilde{r}^{\prime}}} \lesssim\|u\|_{\mathbf{X}^{s, b}}^{3}$. By the choice of $(\tilde{q}, \tilde{r})$ and Lemma (3.2), we see $\|\psi\|_{L_{t}^{\tilde{q}} L_{x}^{\tilde{r}}} \lesssim\|\psi\|_{\mathbf{X}^{0, \frac{1}{2}-\epsilon}}$ for some $\epsilon>0$. Hence we get the desired. The remaining cases $U=|\nabla|^{2-n}(u \nabla \bar{u}) u,|\nabla|^{2-n}\left(|u|^{2}\right) \nabla u,|\nabla|^{2-n}\left(|u|^{2}\right) u$ can be similarly shown using (3.6), (3.5) and Lemma 3.2. This completes the proof of Proposition 3.1 .

Lemma 3.2. Let $q, r \geq 2$ (with possible exception when $n=2$ ). If $\frac{2}{q}+\frac{n}{r} \leq \frac{n}{2}$, then

$$
\|u\|_{L_{t}^{q} L_{x}^{r}} \lesssim\|u\|_{\mathbf{X}^{s, b}}
$$

for $s=s(q, r)=\frac{n}{2}-\frac{2}{q}-\frac{n}{r}$ and any $b>\frac{1}{2}$. If $\frac{2}{q}+\frac{n}{r}>\frac{n}{2}$,

$$
\|u\|_{L_{t}^{q} L_{x}^{r}} \lesssim\|u\|_{\mathbf{X}^{0, b+\epsilon}}
$$

for $b=b(q, r)=\frac{n+2}{4}-\frac{1}{q}-\frac{n}{2 r}$ and any $\epsilon>0$. 
The first follows from the estimates $\left\|e^{i t \Delta} f\right\|_{L_{t}^{q} L_{x}^{r}} \lesssim\|f\|_{\dot{H}^{s(q, r)}}$ and the standard argument (for instance see [22]). Interpolation between the first estimate and the trivial $\|u\|_{L_{t}^{2} L_{x}^{2}} \leq\|u\|_{\mathbf{X}^{0,0}}$ give the second.

By using the Proposition 3.1 and standard fixed point argument in $\mathbf{X}^{1, \frac{1}{2}+\epsilon}$ for $0<\epsilon \ll 1$, we prove the first part of Theorem 1.3 . Here we note that $\mathbf{X}^{1, \frac{1}{2}+\epsilon}\left(J_{T}\right) \hookrightarrow$ $C\left([0, T] ; H^{1}\left(\mathbb{R}^{n}\right)\right)$.

Proof of (1) of Theorem 1.3. We first show the local well-posedness. For this purpose we define a nonlinear functional $\mathcal{N}$ by

$$
\mathcal{N}(u)=\phi(t) e^{i \Delta} u_{0}-i \phi(t / T) \int_{0}^{t} e^{i\left(t-t^{\prime}\right) \Delta}\left[V\left(u\left(t^{\prime}\right)\right) u\left(t^{\prime}\right)\right] d t^{\prime},
$$

where $\phi$ is a fixed smooth cut-off function such that $\phi(t)=1$ if $|t|<1$ and $\phi(t)=0$ if $|t|>2$, and $0<T \leq 1$ is fixed. Then we use the well-known properties of $\mathbf{X}^{s, b}$ (for instance see Proposition 2.2 of [15]);

$$
\left\|\phi(t) e^{i t \Delta} u_{0}\right\|_{\mathbf{X}^{s, b}} \lesssim\left\|u_{0}\right\|_{H^{s}}
$$

for any $s, b$, and

$$
\left\|\int_{0}^{t} e^{i\left(t-t^{\prime}\right) \Delta} F\left(t^{\prime}, x\right) d t^{\prime}\right\|_{\mathbf{X}^{s, b}\left(J_{T}\right)} \lesssim T^{1+b^{\prime}-b}\|F\|_{\mathbf{X}^{s, b^{\prime}\left(J_{T}\right)}},
$$

for $s \in \mathbb{R}$ and $-\frac{1}{2}<b^{\prime} \leq 0,0 \leq b \leq b^{\prime}+1$.

Let us define a complete metric space $B_{T, \rho}$ by

$$
B_{T, \rho}=\left\{u \in \mathbf{X}^{s, \frac{1}{2}+\epsilon}\left(J_{T}\right):\|u\|_{\mathbf{X}^{s, \frac{1}{2}+\epsilon}\left(J_{T}\right)} \leq \rho\right\}
$$

with metric $d$ such that $d(u, v)=\|u-v\|_{\mathbf{X}^{s, \frac{1}{2}+\epsilon}\left(J_{T}\right)}$. From (3.7) and (3.8) with $b=\frac{1}{2}+\epsilon, b^{\prime}=-\frac{1}{2}+\epsilon^{\prime}, \epsilon<\epsilon^{\prime}$ it follows that for any $u \in B_{T, \rho}$

$$
\|\mathcal{N}(u)\|_{\mathbf{X}^{s, \frac{1}{2}+\epsilon}} \lesssim\left\|u_{0}\right\|_{H^{s}}+T^{\epsilon^{\prime}-\epsilon}\|V(u) u\|_{\mathbf{X}^{1,-\frac{1}{2}+\epsilon^{\prime}}} .
$$

If $\epsilon^{\prime}$ is sufficiently small, then we deduce from Proposition 3.1 that

$$
\|\mathcal{N}(u)\|_{\mathbf{X}^{s, \frac{1}{2}+\epsilon}} \lesssim\left\|u_{0}\right\|_{H^{s}}+T^{\epsilon^{\prime}-\epsilon}\|u\|_{\mathbf{X}^{s, \frac{1}{2}+\epsilon}\left(J_{T}\right)}^{3} \lesssim\left\|u_{0}\right\|_{H^{s}}+T^{\epsilon^{\prime}-\epsilon} \rho^{3} .
$$

Choosing $\rho$ and $T$ such that $\rho \geq 2 C\left\|u_{0}\right\|_{H^{s}}$ and $C T^{\epsilon^{\prime}-\epsilon} \rho^{3} \leq \rho / 2$ for some constant $C$, we see that the functional $\mathcal{N}$ is a map from $B_{T, \rho}$ to itself. One can now easily observe that $\mathcal{N}$ is a contraction. In fact, using Proposition 3.1 again, one can easily see that for any $u, v \in B_{T, \rho}$ and for sufficiently small $T$

$$
\begin{aligned}
d(\mathcal{N}(u), \mathcal{N}(v)) & \lesssim T^{\epsilon^{\prime}-\epsilon}\|V(u) u-V(v) v\|_{\mathbf{X}^{s,-\frac{1}{2}+\epsilon^{\prime}\left(J_{T}\right)}} \\
& \lesssim T^{\epsilon^{\prime}-\epsilon}\left(\|u\|_{\mathbf{X}^{s, \frac{1}{2}+\epsilon}\left(J_{T}\right)}+\|v\|_{\mathbf{X}^{s, \frac{1}{2}+\epsilon}\left(J_{T}\right)}\right)^{2} d(u, v) \\
& \lesssim T^{\epsilon^{\prime}-\epsilon} \rho^{2} d(u, v) .
\end{aligned}
$$


Hence a choice of small $T$ makes $\mathcal{N}$ be a contraction map. Therefore there is a unique $u \in \mathbf{X}^{s, \frac{1}{2}+\epsilon}\left(J_{T}\right)$ such that $u(t)=e^{i t \Delta} u_{0}+D(t)$, where

$$
D(t)=-i \int_{0}^{t} e^{i\left(t-t^{\prime}\right) \Delta}\left[V(u) u\left(t^{\prime}\right)\right] d t^{\prime} .
$$

In view of Proposition 3.1 and the estimate (3.8), we have for $s>1 / 2$

$$
\|D\|_{\mathbf{X}^{1, \frac{1}{2}+\epsilon}\left(J_{T}\right)} \lesssim\|V(u) u\|_{\mathbf{X}^{1,-\frac{1}{2}+\epsilon}\left(J_{T}\right)} \lesssim\|u\|_{\mathbf{X}^{s, \frac{1}{2}+\epsilon}\left(J_{T}\right)}<\infty .
$$

Hence the smoothing effect is obtained.

3.2. Power type nonlinearity. Adopting the argument in the proof of Proposition 3.1 and using Theorem 1.1, one can easily get the following.

Corollary 3.3. Let $(q, r)$ be a admissible pair satisfying that $2<r \leq 4$ when $n=3$ and $q, r>2$ when $n \geq 4$. Then for $b>\frac{1}{2}$ and every $0<s<1-2 / r$ there holds

$$
\|u \nabla v\|_{L_{t}^{q / 2} L_{x}^{r / 2}} \lesssim\|u\|_{\mathbf{X}^{s, b}}\|v\|_{\mathbf{X}^{1-s, b}}
$$

The local well-posedness of the Cauchy problem (1.4) with $V(u)=\kappa|u|^{\frac{4}{n}}$ is wellknown in $H^{s}$ space and also in $\mathbf{X}^{s, b}$ space [15]. Hence using Corollary 3.3 and following the lines of argument in [15] we get the proposition.

Proposition 3.4. Let $n \geq 3, s_{n}=\frac{1}{2}$ if $n=3$, and $s_{n}=1-\left(\frac{4}{n}\right)\left(\frac{2}{n}\right)$ if $n \geq 4$. Then for $b>\frac{1}{2}$ and every $s>s_{n}$ there is an $\epsilon>0$ such that

$$
\left\|\nabla\left(|u|^{\frac{4}{n}} u\right)\right\|_{\mathbf{X}^{0,-\frac{1}{2}+\epsilon}} \lesssim\|u\|_{\mathbf{X}^{s, b}}^{\frac{4}{n}+1}
$$

Once this is established, the proof of the second part of Theorem 1.3 is almost same with the case of Hartree type nonlinearity, part (1). Hence we omit the detail.

Proof of Proposition 3.4. Using duality it is enough to show that

$$
\left|\left\langle\psi, \nabla\left(|u|^{\frac{4}{n}} u\right)\right\rangle\right| \lesssim\|\psi\|_{\mathbf{X}^{0, \frac{1}{2}-\epsilon}}\|u\|_{\mathbf{X}^{s, b}}^{\frac{4}{n}+1} .
$$

By direct differentiation the left hand side is bounded by a constant multiple of

$$
\left\langle|\psi|,|u|^{\frac{4}{n}}|\nabla u|\right\rangle \lesssim \sum_{N \geq 1}\left\langle|\psi|,|u|^{\frac{4}{n}}\left|\nabla u_{N}\right|\right\rangle
$$

Here $u_{N}=P_{N} u$ for dyadic $N>1$ and $u_{1}=\widetilde{P}_{1} u$ for the projection operator $\widetilde{P}_{1}$ (recall the notation in introduction). Using Hölder's inequality, we see that for $n=3$

$$
\left\langle|\psi|,|u|^{\frac{4}{n}}\left|\nabla u_{N}\right|\right\rangle \lesssim\|\psi\|_{L_{t}^{q_{1}} L_{x}^{r_{1}}}\left\|u \nabla u_{N}\right\|_{L_{t}^{q / 2} L_{x}^{r / 2}}\|u\|_{L_{t}^{q_{2}} L_{x}^{r_{2}}}^{\frac{1}{2}}
$$

where $\left(1 / q_{1}, 1 / r_{1}\right)+(2 / q, 2 / r)+(1 / 3)\left(1 / q_{2}, 1 / r_{2}\right)=1$, and for $n \geq 4$

$$
\left\langle|\psi|,|u|^{\frac{4}{n}}\left|\nabla u_{N}\right|\right\rangle \lesssim\|\psi\|_{L_{t}^{q_{1}} L_{x}^{r_{1}}}\left\|u \nabla u_{N}\right\|_{L_{t}^{q / 2} L_{x}^{r / 2}}^{\frac{4}{n}}\left\|\nabla u_{N}\right\|_{L_{t}^{q_{2}} L_{x}^{r_{2}}}^{1-\frac{4}{2}},
$$


where $\left(1 / q_{1}, 1 / r_{1}\right)+(4 / n)(2 / q, 2 / r)+(1-4 / n)\left(1 / q_{2}, 1 / r_{2}\right)=1$. We want to choose admissible $(q, r)$ which is arbitrarily close to $\left(\frac{8}{3}, 4\right)$ for $n=3$ and $\left(2, \frac{2 n}{n-2}\right)$ for $n \geq 4$, respectively, and non-admissible pairs $\left(q_{1}, r_{1}\right)$ and $\left(q_{2}, r_{2}\right)$ such that $\left(1 / q_{1}, 1 / r_{1}\right)$ and $\left(1 / q_{2}, 1 / r_{2}\right)$ are slightly above and below the Strichartz line, respectively. More precisely, $\epsilon_{1}>2 / q_{1}+n / r_{1}-n / 2>0$ and $0>2 / q_{2}+n / r_{2}-n / 2>-\epsilon_{2}$ for small $\epsilon_{1}, \epsilon_{2}>0$. With the choices of $\left(q_{1}, r_{1}\right)$ and $\left(q_{2}, r_{2}\right)$, using Lemma 3.2 and Proposition 3.3. we have for $\left|s_{1}\right|<1-\frac{2}{r}$ and $\epsilon>0$

$$
\begin{aligned}
\left\langle|\psi|,|u|^{\frac{4}{n}}\left|\nabla u_{N}\right|\right\rangle & \lesssim\|\psi\|_{\mathbf{X}^{0, \frac{1}{2}-\epsilon}}\|u\|_{\mathbf{X}^{s_{1}, b}}\left\|u_{N}\right\|_{\mathbf{X}^{1-s_{1}, b}}\|u\|_{\mathbf{X}^{\epsilon_{0}, b}}^{\frac{1}{3}} \\
& \lesssim N^{1-s_{1}-s}\|\psi\|_{\mathbf{X}^{0, \frac{1}{2}-\epsilon}}\|u\|_{\mathbf{X}^{s_{1}, b}}\|u\|_{\mathbf{X}^{s, b}}\|u\|_{\mathbf{X}^{s, b}}^{\frac{1}{3}}
\end{aligned}
$$

when $n=3$, and

$$
\begin{aligned}
\left\langle|\psi|,|u|^{\frac{4}{n}}\left|\nabla u_{N}\right|\right\rangle & \lesssim\|\psi\|_{\mathbf{X}^{0, \frac{1}{2}-\epsilon}}\|u\|_{\mathbf{X}^{s_{1}, b}}^{\frac{4}{n}}\left\|u_{N}\right\|_{\mathbf{X}^{1-s_{1}, b}}^{\frac{4}{n}}\left\|\nabla u_{N}\right\|_{\mathbf{X}^{\epsilon_{0}, b}}^{1-\frac{4}{n}} \\
& \lesssim N^{\left(1-s-s_{1} \cdot 4 / n+\epsilon_{0}(1-4 / n)\right)}\|\psi\|_{\mathbf{X}^{0, \frac{1}{2}-\epsilon}}\|u\|_{\mathbf{X}^{s_{1}, b}}^{\frac{4}{n}}\|u\|_{\mathbf{X}^{s, b}}^{\frac{4}{n}}\|u\|_{\mathbf{X}^{s, b}}^{1-\frac{4}{n}}
\end{aligned}
$$

when $n \geq 4$. Therefore, for $s>\max \left(s_{1}, 1-s_{1}\right)$ we get

$$
\left|\left\langle\psi,|u|^{\frac{4}{n}} u\right\rangle\right| \lesssim \sum_{N \geq 1} N^{1-s-s_{1}}\|\psi\|_{\mathbf{X}^{0, \frac{1}{2}-\epsilon}}\|u\|_{\mathbf{X}^{s, b}}^{\frac{4}{3}+1}
$$

when $n=2$, and

$$
\left|\left\langle\psi,|u|^{\frac{4}{n}} u\right\rangle\right| \lesssim \sum_{N \geq 1} N^{\left(1-s-s_{1} \cdot 4 / n+\epsilon_{0}(1-4 / n)\right)}\|\psi\|_{\mathbf{X}^{0, \frac{1}{2}-\epsilon}}\|u\|_{\mathbf{X}^{s, b}}^{\frac{4}{n}+1}
$$

when $n \geq 4$. So we get the desired bound provided $s>1-s_{1}$ when $n=3$ and $s>1-s_{1} \frac{4}{n}$ when $n \geq 4$. We now choose admissible pair $(q, r)$ to be arbitrarily close to $\left(\frac{8}{3}, 4\right)$ when $n=3$ and $\left(2, \frac{2 n}{n-2}\right)$ when $n \geq 4$. Then we get the desired bound for $s>\frac{1}{2}$ when $n=3$, and for $s>1-\frac{8}{n^{2}}$ when $n \geq 4$ because we can choose $s_{1}$ to be arbitrarily close to $\frac{1}{2}$ when $n=3$ and to $\frac{2}{n}$ when $n \geq 4$, and $1-\frac{8}{n^{2}}>\max \left(\frac{2}{n}, 1-\frac{2}{n}\right)$. This completes the proof of Proposition 3.4.

\section{Global Well-posedess of Hartree equations.}

In this section we give the proof Theorem 1.4 which improves the global wellposedness results in [3]. Based on $I$-method, the two main ingredients are the almost energy conservation and almost interaction Morawetz inequality. Our improvement results from the better decay control of these crucial estimates (Proposition 4.1, 4.2), which are obtained by exploiting the trilinear interaction estimate (Proposition 2.6). Since we basically follow the usual steps of I-method ([3, 4, 11]), we do not intend to give all the details of the proof. Instead, we are devoted to proving Proposition 4.1, 4.2 after giving a brief explanation about the overall argument . 
The I-method, introduced by Colliander et.al. [5] to handle low regularity initial data, makes use of a smoothing operator $I$ which regularizes a rough solution up to the regularity level of a conservation law by damping high frequency part. For $0<s<1$ the operator $I: H^{s} \rightarrow H^{1}$ (depending on a parameter $N \gg 1$ ) is defined by

$$
\widehat{I f}(\xi) \equiv m(\xi) \widehat{f}(\xi)
$$

where the multiplier $m(\xi)$ is smooth, radially symmetric, nonincreasing in $|\xi|$ and satisfies

$$
m(\xi)= \begin{cases}1 & |\xi| \leq N \\ \left(\frac{N}{|\xi|}\right)^{1-s} & |\xi| \geq 2 N\end{cases}
$$

When the solution $u$ of (1.4) is in $H^{s}, 0<s<1, E(u)$ may not be finite, but $E(I u)$ is finite. Since $I u$ is not a solution to (1.4), $E(I u)$ is not expected to be conserved. However, it is almost conserved and the deviation can be controlled by $O\left(N^{-\sigma}\right)$, $\sigma>0$ since the operator $I$ gets close to the identity as $N$ increases. In Proposition 4.1 we show that for $p=3 / 2$

$$
E(I u)(T)=E\left(I u_{0}\right)+N^{-p+} \Gamma\left(Z_{I}(T)\right),
$$

where $\Gamma(r)=\sum_{1 \leq i \leq k} O\left(r^{m_{i}}\right)$ for some $k, m_{1}, \ldots, m_{k} \geq 1$ and $Z_{I}(T)$ is the iteration space norm defined by

$$
Z_{I}(T)=\sup _{(q, r): \text { admissible }}\|I\langle\nabla\rangle u\|_{L_{t}^{q} L_{x}^{r}\left(J_{T} \times \mathbb{R}^{n}\right)}
$$

After the Morawetz interaction potential for 3-d NLS was introduced by Colliander et al. [6], it was extended to other dimension [4, 11, 24]. To make use of such estimates (e.g. local in time Morawetz inequality in [11]), the restriction $s>1 / 2$ is inevitable. However, this restriction can be removed by using an inequality for $I u$ ([4]). In fact, it is almost valid in the sense that for some $\theta, p>0$

$$
\|I u\|_{L_{t}^{\frac{4(n-1)}{n}}} \underset{L_{x}^{\frac{2(n-1)}{n-2}}\left(J_{T} \times \mathbb{R}^{n}\right)}{ } \lesssim T^{\theta}\left\|I u_{0}\right\|_{L_{x}^{2}}^{\frac{1}{2}}\|I u\|_{L_{t}^{\infty} \dot{H}_{x}^{\frac{1}{2}}\left(J_{T} \times \mathbb{R}^{n}\right)}^{\frac{n-2}{n-1}}+N^{-p+} \Gamma\left(Z_{I}(T)\right) .
$$

The iteration norm $Z_{I}(T)$ is controlled initially provided that the critical Strichartz norm of $I u$ is small (Lemma 3.1 in [3]). More precisely, there is $\delta>0$ such that if

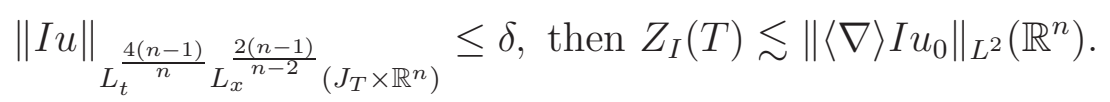

Therefore, once (4.1), (4.2) are obtained, the global well-posedness follows from the usual accounting argument (see Section 5 in [3] or [4] for details). The threshold regularity $s$ is determined by the decay rate $N^{-p+}$. Going over the argument in [3], 
one gets the global well-posedness for $u_{0} \in H^{s}$ as long as $N \gg 1$ can be chosen such that

$$
K N^{\frac{1-s}{s}} \frac{2(n-2)}{n} \sim N^{p-}
$$

for any arbitrarily large $K$. This is possible if $s>\frac{2(n-2)}{(2+p) n-4}$. Consequently, by Proposition 4.1, 4.2 we conclude Theorem 1.4 with $p=3 / 2$.

The rest of this section is devoted to the proofs of the almost energy conservation and almost interaction Morawetz inequality; Proposition 4.1, 4.2.

Proposition 4.1. Let $0<s<1$ and $N \gg 1$. Suppose that $u_{0} \in C_{0}^{\infty}\left(\mathbb{R}^{n}\right)$ and $u$ is the solution to (1.4). Then for any $\epsilon>0$ and $T>0$

$$
\left|E(I u)(T)-E\left(I u_{0}\right)\right| \lesssim N^{-\frac{3}{2}+\epsilon}\left(Z_{I}(T)^{4}+Z_{I}(T)^{6}+Z_{I}(T)^{10}+Z_{I}(T)^{12}\right) .
$$

Now let us consider the $I$-Hartree equation by

$$
i(I u)_{t}+\Delta(I u)=V(I u) I u+[I(V(u) u)-V(I u) I u] \equiv \mathcal{N}_{\text {good }}+\mathcal{N}_{b a d}
$$

where $V(u)=|x|^{-2} *|u|^{2}$. Similarly to the formula (4.31) in [3], we have

$$
\begin{aligned}
-(n-1) \int_{0}^{T} & \iint_{\mathbb{R}^{n} \times \mathbb{R}^{n}} \Delta\left(\frac{1}{|y-x|}\right)|I u(x, t)|^{2}|I u(y, t)|^{2} d x d y d t \\
& +2 \int_{0}^{T} \iint_{\mathbb{R}^{n} \times \mathbb{R}^{n}}|I u(x, t)|^{2} \frac{y-x}{|y-x|} \cdot\left\{\mathcal{N}_{\text {good }}, I u\right\}(y, t) d x d y d t \\
& +2 \int_{0}^{T} \iint_{\mathbb{R}^{n} \times \mathbb{R}^{n}}|I u(x, t)|^{2} \frac{y-x}{|y-x|} \cdot\left\{\mathcal{N}_{\text {bad }}, I u\right\}(y, t) d x d y d t \\
& \lesssim\|I u\|_{L_{t}^{\infty} L_{x}^{2}\left(J_{T} \times \mathbb{R}^{n}\right)}^{2}\|I u\|_{L_{t}^{\infty} \dot{H}^{\frac{1}{2}}\left(J_{T} \times \mathbb{R}^{n}\right)}^{2}
\end{aligned}
$$

Here $\{f, g\}$ denotes $\operatorname{Re}(f \nabla \bar{g}-g \nabla \bar{f})$. Since the second term of (4.3) is positive, it follows from the Hölder's inequality and interpolation (for details see Proposition 4.1 in [3] or Lemma 5.6 in [24]) that

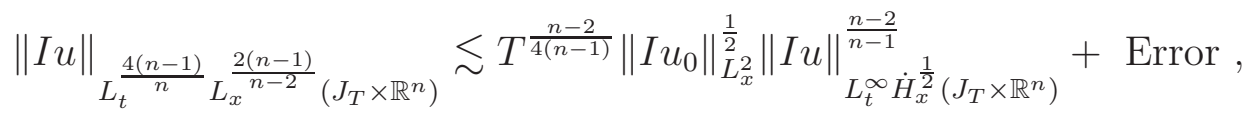

where

$$
\text { Error }=\left.\left|\int_{0}^{T} \iint_{\mathbb{R}^{n} \times \mathbb{R}^{n}}\right| I u(x, t)\right|^{2} \frac{y-x}{|y-x|} \cdot\left\{\mathcal{N}_{b a d}, I u\right\}(y, t) d x d y d t \mid .
$$

Proposition 4.2. Let $0<s<1$ and $N \gg 1$. Suppose that $u_{0} \in C_{0}^{\infty}\left(\mathbb{R}^{n}\right)$ and $u$ is the solution to (1.4). Then for any $\epsilon>0$ and $T>0$.

$$
\text { Error } \lesssim N^{-\frac{3}{2}+\epsilon}\left(Z_{I}(T)^{6}+Z_{I}(T)^{12}\right)
$$


In the whole argument $N$ is assumed to be sufficiently large. So the small frequency part of the solution does not play any significant role. Hence we do not need dyadic decomposition for such portion. Here, we recall that $\widetilde{P}_{1}=i d-\sum_{N>1} P_{N}$. For simplicity, abusing notation, we keep denoting $\widetilde{P}_{1}$ by $P_{1}$. Throughout this section $N_{1}, \ldots, N_{4}$ are dyadic numbers $\geq 1$ and $\sum_{N_{j} \geq 1} P_{N_{j}}=i d$ for $j=1, \ldots, 4$.

4.1. Preliminary estimates. We first show the following inhomogeneous estimate (cf. [8]) for the solutions with localized frequency. For the simplicity of notations we also denote $f_{j}=P_{N_{j}} f, j=1,2,3$.

Lemma 4.3. Let $N_{1}, N_{2}, N_{3}$ be dyadic numbers $\geq 1$ and let $u$ be a smooth solution of $i u_{t}+\Delta u=F$ on $J_{T} \times \mathbb{R}^{n}$ with the initial data $u_{0}$. Then for $\left(u_{1}, u_{2}, u_{3}\right)=$ $\left(P_{N_{1}} u, P_{N_{2}} u, P_{N_{3}} u\right)$ it holds that for any admissible pair $(\tilde{q}, \tilde{r})$

$$
\begin{aligned}
\sup _{\left(\eta_{1}, \eta_{2}, \eta_{3}\right) \in \mathbb{R}^{3 n}} \||\nabla|^{2-n}\left(u_{1}\left(\cdot-\eta_{1}\right)\right. & \left.u_{2}\left(\cdot-\eta_{2}\right)\right) u_{3}\left(\cdot-\eta_{3}\right) \|_{L_{t}^{\tilde{q}^{\prime}} L_{x}^{\tilde{r}^{\prime}}\left(J_{T} \times \mathbb{R}^{n}\right)} \\
& \lesssim C\left(N_{1}, N_{2}, N_{3}\right)\left(\left\|u_{0}\right\|_{L^{2}}^{3}+\|F\|_{L_{t}^{1} L_{x}^{2}\left(J_{T} \times \mathbb{R}^{n}\right)}^{3}\right),
\end{aligned}
$$

where $C\left(N_{1}, N_{2}, N_{3}\right)$ is same as in Proposition 2.6.

We show this by using Proposition 2.6 which works when all $N_{i}>1$. However, due to $P_{1}\left(=\widetilde{P}_{1}\right)$ which has symbol supported in $B(0,2)$, we need to use Corollary 2.8 when one of $N_{i}$ is 1 . By Proposition 2.6 and Corollary 2.8 we get

$$
\|\mathcal{H}(f, g, h)\|_{L_{t}^{\tilde{q}^{\prime}} L_{x}^{\tilde{r}^{\prime}}} \lesssim C\left(N_{1}, N_{2}, N_{3}\right)\|f\|_{L^{2}}\|g\|_{L^{2}}\|h\|_{L^{2}},
$$

for $N_{1}, N_{2}, N_{3}$ dyadic numbers $\geq 1$ and any admissible pair $(\widetilde{q}, \widetilde{r})$.

Proof. By taking $P_{N_{j}}$ to the equation and using Duhamel's formula, we have

$$
\tilde{u}_{j}(t)=e^{i t \Delta}\left(\tilde{u}_{0 j}+\tilde{\mathbf{F}}_{j}(t)\right), j=1,2,3,
$$

where $\tilde{u}_{0 j}=P_{N_{j}}\left(u_{0}\left(\cdot-\eta_{j}\right)\right), \tilde{\mathbf{F}}_{j}(t, x)=-i P_{N_{j}}\left(\int_{0}^{t} \tilde{F}_{j}\left(t^{\prime}\right) d t^{\prime}\right)$ and $\tilde{F}_{j}\left(t^{\prime}\right)=e^{-i t^{\prime} \Delta}\left(F\left(t^{\prime}, \cdot-\right.\right.$ $\left.\eta_{j}\right)$ ). Then we obtain

$$
\left\||\nabla|^{2-n}\left(\tilde{u}_{1} \tilde{u}_{2}\right) \tilde{u}_{3}\right\|_{L_{t}^{\tilde{q}^{\prime}} L_{x}^{\tilde{r}^{\prime}}\left(J_{T} \times \mathbb{R}^{n}\right)} \lesssim \sum_{k=1}^{8} L_{k},
$$

where

$$
\begin{aligned}
& L_{1}=\left\|\mathcal{H}\left(\tilde{u}_{01}, \tilde{u}_{02}, \tilde{u}_{03}\right)\right\|_{L_{t}^{\tilde{q}^{\prime}} L_{x}^{\tilde{r}^{\prime}}\left(J_{T} \times \mathbb{R}^{n}\right)}, L_{2}=\left\|\mathcal{H}\left(\tilde{\mathbf{F}}_{1}, \tilde{u}_{02}, \tilde{u}_{03}\right)\right\|_{L_{t}^{\tilde{q}^{\prime}} L_{x}^{\tilde{r}^{\prime}}\left(J_{T} \times \mathbb{R}^{n}\right)}, \\
& L_{3}=\left\|\mathcal{H}\left(\tilde{u}_{01}, \tilde{\mathbf{F}}_{2}, \tilde{u}_{03}\right)\right\|_{L_{t}^{\tilde{q}^{\prime}} L_{x}^{\tilde{r}^{\prime}}\left(J_{T} \times \mathbb{R}^{n}\right)}, \quad L_{4}=\left\|\mathcal{H}\left(\tilde{u}_{01}, \tilde{u}_{02}, \tilde{\mathbf{F}}_{3}\right)\right\|_{L_{t}^{\tilde{q}^{\prime}} L_{x}^{\tilde{r}^{\prime}}\left(J_{T} \times \mathbb{R}^{n}\right)}, \\
& L_{5}=\left\|\mathcal{H}\left(\tilde{\mathbf{F}}_{1}, \tilde{\mathbf{F}}_{2}, \tilde{u}_{03}\right)\right\|_{L_{t}^{\tilde{q}^{\prime}} L_{x}^{\tilde{r}^{\prime}}\left(J_{T} \times \mathbb{R}^{n}\right)}, \quad L_{6}=\left\|\mathcal{H}\left(\tilde{u}_{01}, \tilde{\mathbf{F}}_{2}, \tilde{\mathbf{F}}_{3}\right)\right\|_{L_{t}^{\tilde{q}^{\prime}} L_{x}^{\tilde{r}^{\prime}}\left(J_{T} \times \mathbb{R}^{n}\right)}, \\
& L_{7}=\left\|\mathcal{H}\left(\tilde{\mathbf{F}}_{1}, \tilde{u}_{02}, \tilde{\mathbf{F}}_{3}\right)\right\|_{L_{t}^{\tilde{q}^{\prime}} L_{x}^{\tilde{r}^{\prime}}\left(J_{T} \times \mathbb{R}^{n}\right)}, \quad L_{8}=\left\|\mathcal{H}\left(\tilde{\mathbf{F}}_{1}, \tilde{\mathbf{F}}_{2}, \tilde{\mathbf{F}}_{3}\right)\right\|_{L_{t}^{\tilde{q}^{\prime}} L_{x}^{\tilde{r}^{\prime}}\left(J_{T} \times \mathbb{R}^{n}\right)},
\end{aligned}
$$


The first term is easily handled by using (4.5). We only consider $L_{8}$. The remaining cases are to be treated similarly. By Minkowski inequality we have

$$
L_{8} \lesssim\left(\int_{J_{T}}\left(\int_{0}^{t} \int_{0}^{t} \int_{0}^{t}\left\|\mathcal{H}\left(\widetilde{F}_{1}\left(t_{1}\right), \widetilde{F}_{2}\left(t_{2}\right), \widetilde{F}_{3}\left(t_{3}\right)\right)\right\|_{L^{\tilde{r}^{\prime}}} d t_{1} d t_{2} d t_{3}\right)^{\tilde{q}^{\prime}} d t\right)^{\frac{1}{\tilde{q}^{\prime}}} .
$$

This above is again bounded by

$$
\int_{J_{T} \times J_{T} \times J_{T}}\left\|\mathcal{H}\left(\widetilde{F}_{1}\left(t_{1}\right), \widetilde{F}_{2}\left(t_{2}\right), \widetilde{F}_{3}\left(t_{3}\right)\right)\right\|_{L_{t}^{\tilde{q}^{\prime}} L_{x}^{\tilde{r}^{\prime}}\left(J_{T} \times \mathbb{R}^{n}\right)} d t_{1} d t_{2} d t_{3} .
$$

Applying (4.5) again, we get $L_{8} \lesssim C\left(N_{1}, N_{2}, N_{3}\right)\|F\|_{L_{t}^{1} L_{x}^{2}\left(J_{T} \times \mathbb{R}^{n}\right)}^{3}$.

Let $\sigma(\xi)$ be infinitely differentiable so that for all $\alpha \in N^{3 n}$ and $\xi=\left(\xi_{1}, \xi_{2}, \xi_{3}\right) \in$ $\mathbb{R}^{3 n}$ there is a constant $c(\alpha)$ with

$$
\left|\partial_{\xi}^{\alpha} \sigma(\xi)\right| \leq c(\alpha)(1+|\xi|)^{-|\alpha|} .
$$

Let us define a multilinear operator $\Lambda$ by

$$
[\Lambda(f, g, h)](x)=\int e^{i x \cdot \xi} \sigma\left(\xi_{1}, \xi_{2}, \xi_{3}\right)\left|\xi_{2}+\xi_{3}\right|^{-(n-2)} \widehat{f}\left(\xi_{1}\right) \widehat{g}\left(\xi_{2}\right) \widehat{h}\left(\xi_{3}\right) d \xi_{1} d \xi_{2} d \xi_{3}
$$

where $\xi=\sum_{i=1}^{3} \xi_{i}$. We notice that $\Lambda(f, g, h)=c f|\nabla|^{2-n}(g h)$ if $\sigma\left(\xi_{1}, \xi_{2}, \xi_{3}\right)=1$. Then we have the following.

Lemma 4.4. Let $\Lambda$ be defined as above and let $u$ be a smooth solution of $i u_{t}+\Delta u=F$ on $J_{T} \times \mathbb{R}^{n}$ with the initial data $u_{0}$. Then for $\left(u_{1}, u_{2}, u_{3}\right)=\left(P_{N_{1}} u, P_{N_{2}} u, P_{N_{3}} u\right)$ it holds that for any admissible pair $(\tilde{q}, \tilde{r})$

$$
\left\|\Lambda\left(u_{1}, u_{2}, u_{3}\right)\right\|_{L_{t}^{\tilde{q}^{\prime}} L_{x}^{\tilde{r}^{\prime}}\left(J_{T} \times \mathbb{R}^{n}\right)} \lesssim C\left(N_{1}, N_{2}, N_{3}\right)\left(\left\|u_{0}\right\|_{L^{2}}^{3}+\|F\|_{L_{t}^{1} L_{x}^{2}\left(J_{T} \times \mathbb{R}^{n}\right)}^{3}\right),
$$

where $C\left(N_{1}, N_{2}, N_{3}\right)$ is same as in Proposition 2.6.

Proof. We choose another Littlewood-Paley projections $\widetilde{P}_{N_{i}}, i=1,2,3$, such that $\widetilde{P}_{N_{i}} P_{N_{i}}=P_{N_{i}}$ and the corresponding cut-off multiplier $\widetilde{\psi}\left(N_{i}^{-1} \xi\right)$ is supported in $A\left(N_{i}\right)$. Then the multilinear operator $\Lambda$ can be rewritten as

$$
\begin{aligned}
& {\left[\Lambda\left(u_{1}, u_{2}, u_{3}\right)\right](x)} \\
& =\int_{\mathbb{R}^{3 n}} e^{i x \cdot \xi} \widetilde{\sigma}_{1,2,3}\left(\xi_{1}, \xi_{2}, \xi_{3}\right)\left|\xi_{2}+\xi_{3}\right|^{-(n-2)} \widehat{u_{3}}\left(\xi_{1}\right) \widehat{u_{2}}\left(\xi_{2}\right) \widehat{u_{1}}\left(\xi_{3}\right) d \xi_{1} d \xi_{2} d \xi_{3} \\
& =c \int_{\eta=\left(\eta_{1}, \eta_{2}, \eta_{3}\right)} \widehat{\widetilde{\sigma}_{1,2,3}}(\eta)\left[u_{1}\left(\cdot-\eta_{1}\right)|\nabla|^{2-n}\left(u_{2}\left(\cdot-\eta_{2}\right) u_{3}\left(\cdot-\eta_{3}\right)\right)\right](x) d \eta,
\end{aligned}
$$

where $\widetilde{\sigma}_{1,2,3}\left(\xi_{1}, \xi_{2}, \xi_{3}\right)=\widetilde{\psi}\left(N_{\sim}^{-1} \xi_{1}\right) \widetilde{\psi}\left(N_{2}^{-1} \xi_{2}\right) \widetilde{\psi}\left(N_{3}^{-1} \xi_{3}\right) \sigma\left(\xi_{1}, \xi_{2}, \xi_{3}\right)$. By the condition (4.6), support condition of $\widetilde{\psi}$ and routine integration by parts, we readily get a 
uniform bound $\left\|\widehat{\widetilde{\sigma}_{1,2,3}}\right\|_{L^{1}\left(\mathbb{R}^{3 n}\right)} \leq C(\alpha)$ with respect to $N_{1}, N_{2}, N_{3}$ for sufficiently large $|\alpha|$. By Minkowski's inequality and Lemma 4.3 we get

$$
\begin{aligned}
& \left\|\Lambda\left(u_{1}, u_{2}, u_{3}\right)\right\|_{L_{t}^{\tilde{q}^{\prime}} L_{x}^{\tilde{r}^{\prime}}\left(J_{T} \times \mathbb{R}^{n}\right)} \\
& \lesssim\left\|\widehat{\widetilde{\sigma}_{1,2,3}}\right\|_{L^{1}\left(\mathbb{R}^{3 n}\right)} \sup _{\eta \in \mathbb{R}^{3 n}}\left\|u_{1}\left(\cdot-\eta_{1}\right)|\nabla|^{2-n}\left(u_{2}\left(\cdot-\eta_{2}\right) u_{3}\left(\cdot-\eta_{3}\right)\right)\right\|_{L_{t}^{\tilde{q}^{\prime}} L_{x}^{\tilde{r}^{\prime}}\left(J_{T} \times \mathbb{R}^{n}\right)} \\
& \lesssim C\left(N_{2}, N_{3}, N_{1}\right)\left(\left\|u_{0}\right\|_{L^{2}}^{3}+\|F\|_{L_{t}^{1} L_{x}^{2}\left(J_{T} \times \mathbb{R}^{n}\right)}^{3}\right) .
\end{aligned}
$$

Since $C\left(N_{2}, N_{3}, N_{1}\right)=C\left(N_{1}, N_{2}, N_{3}\right)$, we obtain the desired.

The operator $I\langle\nabla\rangle$ behaves like $N^{1-s}|\xi|^{s}$ for $|\xi| \gtrsim N$. A Littlewood-Paley theory shows that the Leibniz rule holds for $I\langle\nabla\rangle(f g)$. Thus taking $I\langle\nabla\rangle$ to the equation (1.4), we have

$$
\begin{aligned}
& \|I\langle\nabla\rangle[V(u) u]\|_{L_{t}^{1} L_{x}^{2}\left(J_{T} \times \mathbb{R}^{n}\right)} \\
& \qquad\left(\|u\|_{L_{t}^{3} L_{x}^{3 n-4}\left(J_{T} \times \mathbb{R}^{n}\right)}^{2}\|I\langle\nabla\rangle u\|_{L_{t}^{3} L_{x}^{\frac{6 n}{3 n-4}}\left(J_{T} \times \mathbb{R}^{n}\right)}\right. \\
& \left.\quad+\|u\|_{L_{t}^{\frac{8}{3}} L_{x}^{\frac{4 n}{2 n-3}}\left(J_{T} \times \mathbb{R}^{n}\right)}^{2}\|I\langle\nabla\rangle u\|_{L_{t}^{4} L_{x}^{\frac{2 n}{n-1}}\left(J_{T} \times \mathbb{R}^{n}\right)}\right) \lesssim Z_{I}(T)^{3} .
\end{aligned}
$$

Lemma 4.5. Let $u$ solve $i u_{t}+\Delta u=V(u) u$ with the initial data $u_{0} \in C_{0}^{\infty}$. Then for $\left(u_{1}, u_{2}, u_{3}\right)=\left(P_{N_{1}} u, P_{N_{2}} u, P_{N_{3}} u\right)$ it holds that for any $0<s<1, T>0$ and admissible pair $(\tilde{q}, \tilde{r})$

$$
\left\|\Lambda\left(I\langle\nabla\rangle u_{1}, I\langle\nabla\rangle u_{2}, I\langle\nabla\rangle u_{3}\right)\right\|_{L_{t}^{\tilde{q}^{\prime}} L_{x}^{\tilde{r}^{\prime}}\left(J_{T} \times \mathbb{R}^{n}\right)} \lesssim C\left(N_{1}, N_{2}, N_{3}\right)\left(Z_{I}(T)^{3}+Z_{I}(T)^{9}\right),
$$

where $C\left(N_{1}, N_{2}, N_{3}\right)$ is same as in Proposition 2.6.

Now we are ready to prove the propositions. We first give the proof of Proposition 4.1 .

4.2. Proof of Proposition 4.1. Differentiating the energy $E(I u)(t)$ of $I u$ with respect to time, $\frac{d}{d t} E(I u)(t)=\operatorname{Re} \int_{\mathbb{R}^{n}} \overline{\partial_{t} I u}[V(I u) I u-I(V(u) u)] d x$. Thus we get

$$
E(I u(T))-E(I u(0))=\operatorname{Re} \int_{0}^{T} \int_{\mathbb{R}^{n}} \overline{\partial_{t} I u}[V(I u) I u-I(V(u) u)] d x d t^{\prime} .
$$

We apply the Parseval formula to the right hand side and use the equation (1.4) to get

$$
|E(I u(T))-E(I u(0))| \lesssim E_{a}+E_{b}
$$


where

$$
\begin{aligned}
& E_{a} \equiv\left|\int_{0}^{T} \int_{\sum_{j=1}^{4} \xi_{j}=0}\left(1-\frac{m\left(\xi_{2}+\xi_{3}+\xi_{4}\right)}{m\left(\xi_{2}\right) m\left(\xi_{3}\right) m\left(\xi_{4}\right)}\right)\right| \xi_{2}+\left.\xi_{3}\right|^{-(n-2)} \\
& \times \widehat{\Delta \overline{I u}}\left(\xi_{1}\right) \widehat{\overline{I u}}\left(\xi_{2}\right) \widehat{\operatorname{Iu}}\left(\xi_{3}\right) \widehat{\operatorname{Iu}}\left(\xi_{4}\right) d \xi_{1} d \xi_{2} d \xi_{3} d \xi_{4} d t^{\prime} \mid \\
& E_{b} \equiv\left|\int_{0}^{T} \int_{\sum_{j=1}^{4} \xi_{j}=0}\left(1-\frac{m\left(\xi_{2}+\xi_{3}+\xi_{4}\right)}{m\left(\xi_{2}\right) m\left(\xi_{3}\right) m\left(\xi_{4}\right)}\right)\right| \xi_{2}+\left.\xi_{3}\right|^{-(n-2)} \\
& \times(\widehat{\overline{I(V(u) u})})\left(\xi_{1}\right) \widehat{I u}\left(\xi_{2}\right) \widehat{I u}\left(\xi_{3}\right) \widehat{I u}\left(\xi_{4}\right) d \xi_{1} d \xi_{2} d \xi_{3} d \xi_{4} d t^{\prime} \mid
\end{aligned}
$$

For both $E_{a}$ and $E_{b}$, we break $u$ into $u_{i} \equiv P_{N_{i}} u(i=1,2,3,4)$ and exploit the interaction between Schrödinger waves of different frequency level using Proposition 2.6.

For $E_{a}$ we show that for all $T>0$ and $\epsilon>0$

$$
E_{a} \lesssim N^{-\frac{3}{2}+\epsilon}\left(Z_{I}(T)^{4}+Z_{I}(T)^{10}\right)
$$

It was shown in [3] with $N^{-1+}$. The improvement is actually due to the interaction gain of $\left(N_{j} / N_{k}\right)^{\frac{1}{2}}$ in Lemma 4.5. Let us set

$$
B=B\left(N_{2}, N_{3}, N_{4}\right) \equiv \sup _{\left|\xi_{2}\right| \sim N_{2},\left|\xi_{3}\right| \sim N_{3},\left|\xi_{4}\right| \sim N_{4}}\left|1-\frac{m\left(\xi_{2}+\xi_{3}+\xi_{4}\right)}{m\left(\xi_{2}\right) m\left(\xi_{3}\right) m\left(\xi_{4}\right)}\right| .
$$

By dyadic decomposition and factoring $B\left(N_{2}, N_{3}, N_{4}\right)$ out from the integral in $E_{a}$, we get

$$
\begin{aligned}
E_{a} & \lesssim \sum_{N_{1}, N_{2}, N_{3}, N_{4}} B\left|\int_{0}^{T} \int_{\mathbb{R}^{n}} \mathcal{F}^{-1}\left[\Lambda\left(\Delta \overline{I u_{1}}, \overline{I u_{2}}, I u_{3}\right)\right]\left(\xi_{4}\right) \mathcal{F}\left(I u_{4}\right)\left(\xi_{4}\right) d \xi_{4} d t^{\prime}\right| \\
& =\sum_{N_{1}, N_{2}, N_{3}, N_{4}} B\left|\int_{0}^{T} \int_{\mathbb{R}^{n}}\left[\Lambda\left(\Delta \overline{I u_{1}}, \overline{I u_{2}}, I u_{3}\right)\right](x) I u_{4}(x) d x d t^{\prime}\right|
\end{aligned}
$$

where $\Lambda$ is the multiplier operator as defined by (4.7) with the symbol

$$
\sigma\left(\xi_{1}, \xi_{2}, \xi_{3}\right)=\frac{1}{B\left(N_{2}, N_{3}, N_{4}\right)}\left(1-\frac{m\left(\xi_{2}+\xi_{3}+\xi_{4}\right)}{m\left(\xi_{2}\right) m\left(\xi_{3}\right) m\left(\xi_{4}\right)}\right) .
$$

Note that $\sigma$ satisfies the condition (4.6). Then for (4.12) we need to show

$$
\sum_{N_{1}, N_{2}, N_{3}, N_{4}} B \frac{N_{1}}{\left\langle N_{2}\right\rangle\left\langle N_{3}\right\rangle\left\langle N_{4}\right\rangle} \mathcal{E}_{a}\left(\widetilde{u}_{1}, \widetilde{u}_{2}, \widetilde{u}_{3}, \widetilde{u}_{4}\right) \lesssim N^{-\frac{3}{2}+\epsilon}\left(Z_{I}(T)^{4}+Z_{I}(T)^{10}\right),
$$

where $\widetilde{u}_{1}=N_{1}^{-1} \Delta\langle\nabla\rangle^{-1} u_{1}$ and $\widetilde{u}_{j}=N_{j}\langle\nabla\rangle^{-1} u_{j}, j=2,3,4$, and

$$
\mathcal{E}_{a}\left(w_{1}, w_{2}, w_{3}, w_{4}\right)=\left|\int_{0}^{t} \int_{\mathbb{R}^{n}}\left[\Lambda\left(I\langle\nabla\rangle \overline{w_{1}}, I\langle\nabla\rangle \overline{w_{2}}, I\langle\nabla\rangle w_{3}\right)\right](x) I\langle\nabla\rangle w_{4}(x) d x d t^{\prime}\right| .
$$


Now note that $\mathcal{E}_{a}\left(\widetilde{u}_{1}, \widetilde{u}_{2}, \widetilde{u}_{3}, \widetilde{u}_{4}\right)=\mathcal{E}_{a}\left(\widetilde{\widetilde{u}}_{4}, \widetilde{u}_{2}, \widetilde{u}_{3}, \widetilde{\widetilde{u}}_{1}\right)$. Hence by Hölder's inequality

$$
\mathcal{E}_{a}\left(\widetilde{u}_{1}, \widetilde{u}_{2}, \widetilde{u}_{3}, \widetilde{u}_{4}\right) \leq\left\|\Lambda\left(I\langle\nabla\rangle \widetilde{u}_{4}, I\langle\nabla\rangle \widetilde{u}_{2}, I\langle\nabla\rangle \widetilde{u}_{3}\right)\right\|_{L_{t}^{\tilde{q}^{\prime}} L_{x}^{\tilde{r}^{\prime}}\left(J_{T} \times \mathbb{R}^{n}\right)}\left\|I\langle\nabla\rangle \widetilde{\widetilde{u}}_{1}\right\|_{L_{t}^{\tilde{q}} L_{x}^{\tilde{r}}\left(J_{T} \times \mathbb{R}^{n}\right)} .
$$

Taking admissible $(\tilde{q}, \tilde{r})$ we now apply Corollary 4.5 and using Sobolev imbedding (or Bernstein's inequality) and Hörmander-Mikhlin theorem it follows that

$$
\mathcal{E}_{a}\left(\widetilde{u}_{1}, \widetilde{u}_{2}, \widetilde{u}_{3}, \widetilde{u}_{4}\right) \lesssim C\left(N_{4}, N_{2}, N_{3}\right)\left(Z_{I}(T)^{4}+Z_{I}(T)^{10}\right)
$$

For simplicity we also set

$$
a\left(N_{1}, N_{2}, N_{3}, N_{4}\right) \equiv \frac{B\left(N_{2}, N_{3}, N_{4}\right) N_{1}}{\left\langle N_{2}\right\rangle\left\langle N_{3}\right\rangle\left\langle N_{4}\right\rangle} \times C\left(N_{4}, N_{2}, N_{3}\right) .
$$

Then for (4.12) it is sufficient to show that for all $t \in J_{T}$ and $\epsilon>0$

$$
\sum_{N_{1}, N_{2}, N_{3}, N_{4} \geq 1} a\left(N_{1}, N_{2}, N_{3}, N_{4}\right) \lesssim N^{-\frac{3}{2}+\epsilon} .
$$

Proof of (4.15). Since $B$ and $C\left(N_{2}, N_{3}, N_{4}\right)$ are symmetric on the permutation of $N_{2}, N_{3}, N_{4}$, we may assume

$$
N_{2} \geq N_{3} \geq N_{4}
$$

Then for the proof we consider the sums of the three cases $N \gg N_{2}, N_{2} \gtrsim N \gg$ $N_{3} \geq N_{4}, N_{3} \gtrsim N$, separately.

Case 1. $N \gg N_{2}$. We have $m\left(\xi_{i}\right)=1, i=2,3,4$, and $m\left(\xi_{1}\right)=1$ since $\sum_{i=1}^{4} \xi_{i}=0$. So, the symbol $\left(1-\frac{m\left(\xi_{1}\right)}{m\left(\xi_{2}\right) m\left(\xi_{3}\right) m\left(\xi_{4}\right)}\right)=0$. Hence

$$
B\left(N_{2}, N_{3}, N_{4}\right)=0 .
$$

Case 2. $N_{2} \gtrsim N \gg N_{3} \geq N_{4}$. Since $\sum_{i=1}^{4} \xi_{i}=0$, we have $N_{1} \sim N_{2}$. By the mean value theorem,

$$
\left|1-\frac{m\left(\xi_{1}\right)}{m\left(\xi_{2}\right) m\left(\xi_{3}\right) m\left(\xi_{4}\right)}\right|=\left|\frac{m\left(\xi_{2}\right)-m\left(\xi_{2}+\xi_{3}+\xi_{4}\right)}{m\left(\xi_{2}\right)}\right| \lesssim \frac{\left|\nabla m\left(\xi_{2}\right) \cdot\left(\xi_{3}+\xi_{4}\right)\right|}{m\left(\xi_{2}\right)} \lesssim \frac{N_{3}}{N_{2}} .
$$

Since $C\left(N_{2}, N_{3}, N_{4}\right)=\left(N_{4} / N_{2}\right)^{\frac{1}{2}}$, we thus have

$$
a \lesssim \frac{1}{N_{2} N_{4}}\left(\frac{N_{4}}{N_{2}}\right)^{\frac{1}{2}}
$$

Taking sum in the order of $N_{2}, N_{3}, N_{4}$, we get

$$
\sum_{N_{1} \sim N_{2} \geq N \gg N_{3} \geq N_{4}} a \lesssim N^{-\frac{3}{2}} \ln N .
$$

Case 3. $N_{3} \gtrsim N$. For this we need only to consider two subcases $N_{1} \sim N_{2}$ and $N_{2} \gg N_{1}$ since the case $N_{1} \gg N_{2}$ cannot happen by the condition $\sum_{i} \xi_{i}=0$. 
Case 3-1. $N_{3} \gtrsim N, N_{1} \sim N_{2}$. In this case, we have the bound

$$
\left|1-\frac{m\left(\xi_{1}\right)}{m\left(\xi_{2}\right) m\left(\xi_{3}\right) m\left(\xi_{4}\right)}\right| \frac{N_{1}}{N_{2} N_{3} N_{4}} \lesssim \frac{1}{N_{3} m\left(\xi_{3}\right) N_{4} m\left(\xi_{4}\right)}
$$

since $0<m\left(\xi_{i}\right) \leq 1$. Then we consider two possible cases $N_{1} \sim N_{2} \geq N_{3} \geq N_{4} \gtrsim N$, $N_{1} \sim N_{2} \geq N_{3} \geq N \gg N_{4}$, separately. When $N_{1} \sim N_{2} \geq N_{3} \geq N_{4} \gtrsim N$, we have

$$
a \lesssim \sup _{\left|\xi_{3}\right| \sim N_{3},\left|\xi_{4}\right| \sim N_{4}} \frac{1}{N_{3} m\left(\xi_{3}\right) N_{4} m\left(\xi_{4}\right)}\left(\frac{N_{4}}{N_{2}}\right)^{\frac{1}{2}} \lesssim \frac{1}{N^{2-2 s} N_{3}^{s} N_{4}^{s}}\left(\frac{N_{4}}{N_{2}}\right)^{\frac{1}{2}} .
$$

Hence, summation in the order of $N_{2}, N_{3}, N_{4}$ gives

$$
\sum_{N_{1} \sim N_{2} \geq N_{3} \geq N_{4} \geq N} a \lesssim N^{-2} .
$$

For the case $N_{1} \sim N_{2} \geq N_{3} \geq N \gg N_{4}$, it follows from the fact $m\left(\xi_{4}\right)=1$ that

$$
a \lesssim \sup _{\left|\xi_{3}\right| \sim N_{3},\left|\xi_{4}\right| \sim N_{4}} \frac{1}{N_{3} m\left(\xi_{3}\right) N_{4}}\left(\frac{N_{4}}{N_{2}}\right)^{\frac{1}{2}} \lesssim \frac{1}{N^{1-s} N_{3}^{s} N_{4}}\left(\frac{N_{4}}{N_{2}}\right)^{\frac{1}{2}} .
$$

Summing $N_{2}, N_{3}, N_{4}$, successively, we have acceptable bound

$$
\sum_{N_{1} \sim N_{2} \geq N_{3} \geq N_{4} \gtrsim N} a \lesssim N^{-\frac{3}{2}}
$$

Case 3-2. $N_{3} \gtrsim N, N_{2} \gg N_{1}$. We have $N_{2} \sim N_{3}$ from $\sum_{i=1}^{4} \xi_{i}=0$. Since $m\left(\xi_{1}\right) \geq m\left(\xi_{2}\right)$, we get

$$
\left|1-\frac{m\left(\xi_{2}+\xi_{3}+\xi_{4}\right)}{m\left(\xi_{2}\right) m\left(\xi_{3}\right) m\left(\xi_{4}\right)}\right| \frac{N_{1}}{N_{2} N_{3} N_{4}} \lesssim \frac{m\left(\xi_{1}\right)}{m\left(\xi_{2}\right)^{2} m\left(\xi_{4}\right)} \frac{N_{1}}{N_{2}^{2} N_{4}} .
$$

We handle the cases $N_{1} \gtrsim N$ and $N_{1} \leq N$, separately.

If $N_{1} \gtrsim N$, we have three possible cases; $N_{2} \sim N_{3} \geq N_{4} \geq N_{1} \geq N, N_{2} \sim N_{3} \geq$ $N_{1} \geq N_{4} \geq N, N_{2} \sim N_{3} \gg N_{1} \gtrsim N \geq N_{4}$. When $N_{2} \sim N_{3} \geq N_{4} \geq N_{1} \geq N$, using (4.16) we get

$$
a \lesssim \frac{N_{1}^{s}}{N^{2-2 s} N_{2}^{2 s} N_{4}^{s}}\left(\frac{N_{4}}{N_{2}}\right)^{\frac{1}{2}}
$$

Summing in the order of $N_{2}, N_{4}, N_{1}$, we have $\sum_{N_{2} \sim N_{3} \geq N_{4} \geq N_{1} \geq N} a \lesssim N^{-2}$, which is acceptable. Similarly, when $N_{2} \sim N_{3} \geq N_{1} \geq N_{4} \geq N$, it follows

$$
a \lesssim \frac{N_{1}^{s}}{N^{2-2 s} N_{2}^{2 s} N_{4}^{s}}\left(\frac{N_{4}}{N_{2}}\right)^{\frac{1}{2}} .
$$

Then we get $\sum_{N_{2} \sim N_{3} \geq N_{1} \geq N_{4} \geq N} a \lesssim N^{-2}$ by summing in $N_{2}, N_{1}, N_{4}$, successively. Finally, when $N_{2} \sim N_{3} \gg N_{1} \gtrsim N \geq N_{4}$, we have

$$
a \lesssim \frac{N_{1}^{s}}{N^{1-s} N_{2}^{2 s} N_{4}}\left(\frac{N_{4}}{N_{2}}\right)^{\frac{1}{2}} .
$$


So summation gives $\sum_{N_{2} \sim N_{3} \gg N_{1} \gtrsim N \geq N_{4}} a \lesssim N^{-\frac{3}{2}}$.

Now we turn to the case $N_{1} \leq N$. We again have three possible cases; $N_{2} \sim N_{3} \geq$ $N_{4} \geq N \geq N_{1}, N_{2} \sim N_{3} \geq N \geq N_{4} \geq N_{1}, N_{2} \sim N_{3} \geq N \geq N_{1} \geq N_{4}$. Firstly, when $N_{2} \sim N_{3} \geq N_{4} \geq N \geq N_{1}$, we have

$$
a \lesssim \frac{N_{1}}{N^{3-3 s} N_{2}^{2 s} N_{4}^{s}}\left(\frac{N_{4}}{N_{2}}\right)^{\frac{1}{2}}
$$

which gives acceptable bound $\sum_{N_{2} \sim N_{3} \geq N_{4} \geq N \geq N_{1}} a \lesssim N^{-2}$. For the case $N_{2} \sim N_{3} \geq$ $N \geq N_{4} \geq N_{1}$ it follows that

$$
a \lesssim \frac{N_{1}}{N^{2-2 s} N_{2}^{2 s} N_{4}}\left(\frac{N_{4}}{N_{2}}\right)^{\frac{1}{2}}
$$

So, we have $\sum_{N_{2} \sim N_{3} \geq N \geq N_{4} \geq N_{1}} a \lesssim N^{-2}$. Finally when $N_{2} \sim N_{3} \geq N \geq N_{1} \geq N_{4}$, we have

$$
a \lesssim \frac{N_{1}}{N^{2-2 s} N_{2}^{2 s} N_{4}}\left(\frac{N_{4}}{N_{2}}\right)^{\frac{1}{2}}
$$

Summation gives the bound $\sum_{N_{2} \sim N_{3} \geq N \geq N_{1} \geq N_{4}} a \lesssim N^{-\frac{3}{2}}$. Thus we conclude the proof of (4.15).

Now we turn to $E_{b}$ and claim

$$
E_{b} \lesssim N^{-\frac{3}{2}+\epsilon}\left(Z_{I}(T)^{6}+Z_{I}(T)^{12}\right) .
$$

Decomposing the integral in (4.11) dyadically, factoring $B\left(N_{2}, N_{3}, N_{4}\right)$ out and using Plancherel's formula as before, we see that

$$
\begin{aligned}
E_{b} & \leq \sum_{N_{1}, N_{2}, N_{3}, N_{4}} B\left|\int_{0}^{T} \int_{\mathbb{R}^{n}} \mathcal{F}^{-1}\left[\Lambda\left(\overline{I u_{2}}, I u_{3}, I u_{4}\right)\right]\left(\xi_{1}\right) \mathcal{F}\left(P_{N_{1}} I(V(u) \bar{u})\right)\left(\xi_{1}\right) d \xi_{1} d t^{\prime}\right| \\
& =\sum_{N_{1}, N_{2}, N_{3}, N_{4}} B\left|\int_{0}^{T} \int_{\mathbb{R}^{n}}\left[\Lambda\left(I \overline{u_{2}}, I u_{3}, I u_{4}\right)\right](x) P_{N_{1}} I(V(u) \bar{u})(x) d x d t^{\prime}\right| .
\end{aligned}
$$

Hence, we see

$$
E_{b} \lesssim \sum_{N_{1}, N_{2}, N_{3}, N_{4}} \frac{B}{\left\langle N_{2}\right\rangle\left\langle N_{3}\right\rangle\left\langle N_{4}\right\rangle} \times \mathcal{E}_{b}\left(u, \tilde{u}_{2}, \tilde{u}_{3}, \tilde{u}_{4}\right)
$$

where $\mathcal{E}_{b}$ is defined by

$\mathcal{E}_{b}\left(u, w_{2}, w_{3}, w_{4}\right) \equiv\left|\int_{0}^{T} \int_{\mathbb{R}^{n}}\left[\Lambda\left(I\langle\nabla\rangle \overline{w_{2}}, I\langle\nabla\rangle w_{3}, I\langle\nabla\rangle w_{4}\right)\right](x) P_{N_{1}} I(V(u) \bar{u})(x) d x d t^{\prime}\right|$.

Here $\tilde{u}_{j}$ are defined by the same way as for $\mathcal{E}_{a}$. We need the following lemma to get a control of $\mathcal{E}_{b}$. 
Lemma 4.6. Let $u$ be a smooth solution of $i u_{t}=\Delta u+V(u) u$ with initial data $u_{0}$ on $J_{T} \times \mathbb{R}^{n}$. Then there holds

$$
\mathcal{E}_{b} \lesssim C\left(N_{2}, N_{3}, N_{4}\right) N_{1}\left(Z_{I}(T)^{6}+Z_{I}(T)^{12}\right)
$$

Proof. For any admissible pair $(q, r)$, the Hölder's inequality yields

$$
\mathcal{E}_{b} \leq\left\|\Lambda\left(I\langle\nabla\rangle \tilde{\tilde{u}}_{2}, I\langle\nabla\rangle \tilde{u}_{3}, I\langle\nabla\rangle \tilde{u}_{4}\right)\right\|_{L_{t}^{q^{\prime}} L_{x}^{r^{\prime}}\left(J_{T} \times \mathbb{R}^{n}\right)}\left\|P_{N_{1}} I(V(u) \bar{u})\right\|_{L_{t}^{q} L_{x}^{r}\left(J_{T} \times \mathbb{R}^{n}\right)} .
$$

Applying Lemma 4.5 and Hörmander-Mikhlin theorem we have

$$
\mathcal{E}_{b} \lesssim C\left(N_{2}, N_{3}, N_{4}\right)\left(Z_{I}(T)^{3}+Z_{I}(T)^{9}\right)\left\|P_{N_{1}} I(V(u) \bar{u})\right\|_{L_{t}^{q} L_{x}^{r}\left(J_{T} \times \mathbb{R}^{n}\right)} .
$$

Then Lemma 4.6 is the consequence of the estimate

$$
\left\|P_{N_{1}} I(V(u) \bar{u})\right\|_{L_{t}^{q} L_{x}^{r}\left(J_{T} \times \mathbb{R}^{n}\right)} \lesssim N_{1}\left(Z_{I}(T)\right)^{3}
$$

for admissible $(q, r)$ with $2 \leq q \leq 4$ if $n=3$ and $2 \leq q \leq \infty$ if $n \geq 4$.

In fact, using Bernstein's inequality and Hörmander-Mikhlin theorem, we see that for $r \geq \tilde{r}$

$$
\left\|P_{N_{1}} I(V(u) \bar{u})\right\|_{L_{x}^{r}} \lesssim N_{1}^{\frac{n}{\tilde{r}}-\frac{n}{r}}\left\|P_{N_{1}} I(V(u) \bar{u})\right\|_{L_{x}^{\tilde{r}}} \lesssim N_{1}^{\frac{n}{\tilde{r}}-\frac{n}{r}-1}\|I\langle\nabla\rangle(V(u) \bar{u})\|_{L_{x}^{\tilde{r}}}
$$

From Leibniz rule for the operator $I\langle\nabla\rangle$ and Hölder's inequality with $1 / \tilde{r}=1 / r_{1}+$ $1 / r_{2}$ we bound $\|I\langle\nabla\rangle(V(u) \bar{u})\|_{L_{x}^{\tilde{r}}}$ by

$$
\left\||x|^{-2} *\left(I\langle\nabla\rangle|u|^{2}\right)\right\|_{L_{x}^{r_{1}}}\|u\|_{L_{x}^{r_{2}}}+\left\||x|^{-2} *|u|^{2}\right\|_{L_{x}^{r_{1}}\|I\langle\nabla\rangle u\|_{L_{x}^{r_{2}}} .}
$$

It follows from the fractional integration that $\|I\langle\nabla\rangle(V(u) u)\|_{L_{x}^{\tilde{r}}} \lesssim\|I\langle\nabla\rangle u\|_{L_{x}^{r_{2}}}^{3}$ for $1 / r_{1}=2 / r_{2}-(n-2) / n$ and $\frac{n}{n-1}<r_{2}<\frac{2 n}{n-2}$. Since $\tilde{r} \geq 1$, the equation $1 / \tilde{r}=$ $3 / r_{2}-1+2 / n$ also implies $r_{2} \geq 3 n /(2 n-2)$. Combining this with (4.20) we get

$$
\left\|P_{N_{1}} I(V(u) u)\right\|_{L_{t}^{q} L_{x}^{r}\left(J_{T} \times \mathbb{R}^{n}\right)} \lesssim N_{1}^{n\left(\frac{3}{r_{2}}-\frac{1}{r}-1+\frac{2}{n}\right)-1}\|I\langle\nabla\rangle u\|_{L_{t}^{3 q} L_{x}^{r_{2}}\left(J_{T} \times \mathbb{R}^{n}\right)} .
$$

If $(q, r)$ and $\left(3 q, r_{2}\right)$ are admissible, then $n\left(\frac{3}{r_{2}}-\frac{1}{r}-1+\frac{2}{n}\right)-1=1$. The admissibility and range of $r_{2}$ ensure that $2 \leq q \leq 4$ if $n=3$ and $2 \leq q \leq \infty$ if $n \geq 4$. This proves (4.19).

Using (4.18), Lemma 4.6 and the Hörmander-Mikhlin theorem, we have

$$
E_{b} \lesssim \sum_{N_{1}, N_{2}, N_{3}, N_{4}} B \frac{\left\langle N_{1}\right\rangle}{\left\langle N_{2}\right\rangle\left\langle N_{3}\right\rangle\left\langle N_{4}\right\rangle} C\left(N_{2}, N_{3}, N_{4}\right)\left(Z_{I}(T)^{6}+Z_{I}(T)^{12}\right)
$$

Then from (4.15) we see $\sum_{N_{1}, N_{2}, N_{3}, N_{4}} B \frac{\left\langle N_{1}\right\rangle}{\left\langle N_{2}\right\rangle\left\langle N_{3}\right\rangle\left\langle N_{4}\right\rangle} C \lesssim N^{-\frac{3}{2}+\epsilon}$. Therefore we get the desired (4.17). This completes the proof of Proposition 4.1. 
4.3. Proof of Proposition 4.2. We recall that $\mathcal{N}_{\text {bad }}=I(V(u) u)-V(I u) I u$. Then by Hölder's inequality we get

$$
\begin{aligned}
\text { Error } & =\left.\left|\int_{0}^{T} \iint_{\mathbb{R}^{n} \times \mathbb{R}^{n}}\right| I u(x, t)\right|^{2} \frac{y-x}{|y-x|} \cdot\left(\mathcal{N}_{b a d} \nabla \overline{I u}-I u \nabla \overline{\mathcal{N}_{b a d}}\right)(y, t) d x d y d t \mid \\
& \leq\left(\int_{0}^{T} \int_{\mathbb{R}^{n}}\left(\left|\mathcal{N}_{b a d}\right| \cdot|\nabla I u|+\left|\nabla \mathcal{N}_{b a d}\right| \cdot|I u|\right) d y d t\right)\|I u\|_{L_{t}^{\infty} L_{x}^{2}\left(J_{T} \times \mathbb{R}^{n}\right)}^{2} \\
& \lesssim\left\|\langle\nabla\rangle \mathcal{N}_{b a d}\right\|_{L_{t}^{\tilde{q}^{\prime}} L_{x}^{\tilde{r}^{\prime}}\left(J_{T} \times \mathbb{R}^{n}\right)}\|\langle\nabla\rangle I u\|_{L_{t}^{\tilde{q}} L_{x}^{\tilde{r}}}\|I u\|_{L_{t}^{\infty} L_{x}^{2}\left(J_{T} \times \mathbb{R}^{n}\right)}^{2} \\
& \lesssim\|\langle\nabla\rangle[I(V(u) u)-V(I u) I u]\|_{L_{t}^{\tilde{q}^{\prime}} L_{x}^{\tilde{r}^{\prime}}\left(J_{T} \times \mathbb{R}^{n}\right)}\left(Z_{I}(T)\right)^{3} .
\end{aligned}
$$

Hence the proof of Proposition 4.2 is reduced to showing that

$$
\|\langle\nabla\rangle[I(V(u) u)-V(I u) I u]\|_{L_{t}^{\tilde{q}^{\prime}} L_{x}^{\tilde{r}^{\prime}}\left(J_{T} \times \mathbb{R}^{n}\right)} \lesssim N^{-\frac{3}{2}}\left(Z_{I}(T)\right)^{3} .
$$

For any fixed $\psi \in L_{t}^{\tilde{q}^{\prime}} L_{x}^{\tilde{r}^{\prime}}\left(J_{T} \times \mathbb{R}^{n}\right)$ we set

$$
E_{c}=\left|\int_{0}^{T} \int_{\mathbb{R}^{n}}\langle\nabla\rangle[I(V(u) u)-V(I u) I u] \bar{\psi} d x d t\right| .
$$

Then by duality it suffices to show that for $\epsilon>0$

$$
E_{c} \lesssim N^{-\frac{3}{2}+\epsilon}\left(Z_{I}(T)^{3}+Z_{I}(T)^{9}\right)\|\psi\|_{L_{t}^{\tilde{q}} L_{x}^{\tilde{r}}\left(J_{T} \times \mathbb{R}^{n}\right)}
$$

We now follow the similar lines argument as in the proof of the Proposition 4.1. By Plancherel's theorem we have

$$
E_{c} \sim\left|\int_{0}^{T} \int_{\sum_{j=1}^{4} \xi_{i}=0} \tilde{\sigma}\left(\xi_{2}, \xi_{3}, \xi_{4}\right)\right| \xi_{2}+\left.\xi_{3}\right|^{-(n-2)} \widehat{\overline{\operatorname{Iu}}}\left(\xi_{2}\right) \widehat{\operatorname{Iu}}\left(\xi_{3}\right) \widehat{\operatorname{Iu}}\left(\xi_{4}\right) \widehat{\bar{\psi}}\left(\xi_{1}\right) d \xi d t \mid,
$$

where

$$
\widetilde{\sigma}\left(\xi_{2}, \xi_{3}, \xi_{4}\right)=\left\langle\xi_{2}+\xi_{3}+\xi_{4}\right\rangle\left(1-\frac{m\left(\xi_{2}+\xi_{3}+\xi_{4}\right)}{m\left(\xi_{2}\right) m\left(\xi_{3}\right) m\left(\xi_{4}\right)}\right) .
$$

We decompose $u_{1}, u_{2}, u_{3}$ and $\psi$ into the sum of dyadic pieces $u_{j}=P_{N_{j}} u(j=1,2,3,4)$ and $\psi_{1}=P_{N_{1}} \psi$. Let us define the maximum of $|\widetilde{\sigma}|$ on each dyadic piece by

$$
\widetilde{B}=\widetilde{B}\left(N_{2}, N_{3}, N_{4}\right) \equiv \sup _{\left|\xi_{2}\right| \sim N_{2},\left|\xi_{3}\right| \sim N_{3},\left|\xi_{4}\right| \sim N_{4}}\left|\widetilde{\sigma}\left(\xi_{2}, \xi_{3}, \xi_{4}\right)\right|
$$

We now set $\sigma\left(\xi_{2}, \xi_{3}, \xi_{4}\right)=\widetilde{B}^{-1} \widetilde{\sigma}\left(\xi_{2}, \xi_{3}, \xi_{4}\right)$ and define the multilinear operator $\Lambda$ to be as in (4.7) with the symbol $\sigma$. Then

$$
E_{c} \lesssim \sum_{N_{1}, N_{2}, N_{3}, N_{4}}\left|\int_{0}^{T} \int_{\mathbb{R}^{n}}\left[\Lambda\left(\overline{I u_{2}}, I u_{3}, I u_{4}\right)\right](x) \bar{\psi}_{1}(x) d x d t\right|
$$

Hence, as before we see

$$
E_{c} \lesssim \sum_{N_{1}, N_{2}, N_{3}, N_{4}} \frac{\widetilde{B}}{\left\langle N_{2}\right\rangle\left\langle N_{3}\right\rangle\left\langle N_{4}\right\rangle} \times \mathcal{E}_{c}\left(u, \tilde{u}_{2}, \tilde{u}_{3}, \tilde{u}_{4}\right)
$$


where $\widetilde{u}_{j}=\left\langle N_{j}\right\rangle\langle\nabla\rangle^{-1} u_{j}, j=2,3,4$ and $\mathcal{E}_{c}$ is defined by

$$
\mathcal{E}_{c}\left(\psi_{1}, w_{2}, w_{3}, w_{4}\right)=\left|\int_{0}^{T} \int_{\mathbb{R}^{n}}\left[\Lambda\left(I\langle\nabla\rangle \overline{w_{2}}, I\langle\nabla\rangle w_{3}, I\langle\nabla\rangle w_{4}\right)\right](x) \bar{\psi}_{1}(x) d x d t^{\prime}\right| .
$$

Using Hölder's inequality and Lemma 4.5 as before, we get $\mathcal{E}_{c} \lesssim C\left(N_{2}, N_{3}, N_{4}\right)\left(Z_{I}(T)^{3}+\right.$

$\left.Z_{I}(T)^{9}\right)\|\psi\|_{L_{t}^{\widetilde{q}} L_{x}^{\widetilde{r}}\left(J_{T} \times \mathbb{R}^{n}\right)}$. Then by this and (4.22) the proof of (4.21) is reduced to showing that for $\epsilon>0$

$$
\sum_{N_{1}, N_{2}, N_{3}, N_{4}} \frac{\widetilde{B}\left(N_{2}, N_{3}, N_{4}\right)}{\left\langle N_{2}\right\rangle\left\langle N_{3}\right\rangle\left\langle N_{4}\right\rangle} \times C\left(N_{2}, N_{3}, N_{4}\right) \lesssim N^{-\frac{3}{2}+\epsilon} .
$$

Finally notice that $\widetilde{B} \sim B N_{1}$, where $B$ is the same upper bound appearing in the estimates of $E_{a}$ and $E_{b}$. Then we get the desired bound from (4.15). This completes the proof of Proposition 4.2 .

\section{APPENDIX}

Wave packet decomposition. For a fixed $\lambda \gg 1$, let us define the spatial and frequency grids $\mathcal{Y}, \mathcal{V}$, by

$$
\mathcal{Y}=\lambda^{1 / 2} \mathbb{Z}^{n}, \mathcal{V}=\lambda^{-1 / 2} \mathbb{Z}^{n} \cap Q(2),
$$

respectively. For each $(y, v) \in \mathcal{Y} \times \mathcal{V}$, we associate a tube $T_{y, v}$ given by

$$
T_{y, v}=\left\{(x, t) \in \mathbb{R}^{n} \times \mathbb{R}:|t| \leq 4 \lambda,|x-(y+2 t v)| \leq \lambda^{1 / 2}\right\} .
$$

Obviously $T_{y, v}$ meets $(y, 0)$ and its major direction is parallel to $(2 v, 1)$. Let us denote by $\mathcal{T}(\lambda)$ the collection of these cubes. Conversely for a given $T=T_{y, v} \in \mathcal{T}(\lambda)$, we set

$$
y_{T}=y, v_{T}=v .
$$

Let $\eta$ be the function satisfying supp $\widehat{\eta} \subset Q(2)$ and $\sum_{k \in \mathbb{Z}^{n}} \eta(\cdot-k)=1$. Let $\psi \in C_{0}^{\infty}(B(0,1))$ with $\sum_{k \in \mathbb{Z}^{n}} \psi(\cdot-k)=1$. For $T \in \mathcal{T}(\lambda)$ we also set

$$
f_{T}(x)=\eta\left(\frac{x-y_{T}}{\lambda^{1 / 2}}\right) \mathcal{F}^{-1}\left[\widehat{f} \psi\left(\lambda^{1 / 2}\left(\cdot-v_{T}\right)\right)\right] .
$$

Then it is obvious that

$$
e^{i t \Delta} f=\sum_{T \in \mathcal{T}(\lambda)} e^{i t \Delta} f_{T}
$$

provided $\widehat{f}$ is supported in $Q(1)$. Then by routine integration by parts one can see that $e^{i t \Delta} f_{T}$ is essentially supported in $T$. More precisely, for any $\delta>0$ there is a $C=C(M, \delta)$ such that

$$
\left|e^{i t \Delta} f(x)\right| \leq C \lambda^{-M}\|f\|_{L^{2}} \text { if }(x, t) \notin \lambda^{\delta} T .
$$

For the detail of the wave packet decomposition see [21] (also see [16]). For the proof of Proposition 2.4, we use the following estimates due to Tao [21]. 
Lemma 4.7 (Relation $\sim$ between wave packets and $b$ ). Let $1 \ll \lambda, 0<\delta \ll 1$ and $\{b\}$ be the collection of the cubes $b$ of side length $\sim \lambda^{1-\delta}$ partitioning $Q(\lambda) \times(-\lambda, \lambda)$. Suppose that $f, g \in L^{2}$ with $\widehat{f}, \widehat{g}$ supported in $Q(3 / 2)$ and they are decomposed at scale $\lambda$ such that

$$
f=\sum_{T \in \mathcal{T}(\lambda)} f_{T}, \quad g=\sum_{T \in \mathcal{T}(\lambda)} g_{T}
$$

Then if $\operatorname{dist}(\operatorname{supp} \widehat{f}$, supp $\widehat{g}) \sim 1$, then there is a relation $\sim$ between tubes $T \in \mathcal{T}(\lambda)$ and cubes $b \in\{b\}$ such that for any $\epsilon>0$,

$$
\sum_{b}\left\|\sum_{T \sim b} f_{T}\right\|_{L^{2}}^{2} \leq C \lambda^{\epsilon}\|f\|_{L^{2}}^{2}, \quad \sum_{b}\left\|\sum_{T \sim b} g_{T}\right\|_{L^{2}}^{2} \leq C \lambda^{\epsilon}\|g\|_{L^{2}}^{2}
$$

and for any $b$ and $\epsilon>0$,

$$
\left\|\sum_{T \not b \text { or } T^{\prime} \not b} e^{i t \Delta} f_{T} e^{i t \Delta} g_{T^{\prime}}\right\|_{L^{2}(b)} \leq C \lambda^{\epsilon} \lambda^{c \delta-(n-1) / 4}\|f\|_{L^{2}}\|g\|_{L^{2}}
$$

with $c$ independent of $\delta, \epsilon$.

\section{REFERENCES}

[1] J. Bourgain, Refinements of Strichartz' inequality and applications to $2 D$-NLS with critical nonlinearity, Int. Math. Res. Not. 5 (1998) 253-283.

[2] T. Cazenave, Semilinear Schrödinger equations, Courant Lecture Notes in Mathematics, 10, New York University, Courant Institute of Mathematical Sciences, New York, 2003.

[3] M. Chae and S. Kwon, Global well-posedness for the $L^{2}$ critical Hartree eqaution on $\mathbb{R}^{n}, n \geq 3$, to appear in Commun. Pure Appl. Anal.

[4] J. Colliander, M. Grillakis and N. Tzirakis, Improved interaction Morawetz inequalites for the cubic nonlinear Schrödinger equaton in 2d, Int. Math. Res. Not. 23 (2007), Art. ID mm090, $30 \mathrm{pp}$.

[5] J. Colliander, M. Keel, G. Staffilani, H. Takaoka and T. Tao, Almost conservation laws and global rough solutions to a nonlinear Schrödinger equation, Math. Res. Letters 9 (2002), 1-24.

[6] J. Colliander, M. Keel, G. Staffilani, H. Takaoka and T. Tao, Global existence and scattering for rough solutions of nonlinear Schrödinger equation on $\mathbb{R}^{3}$, Commun. Pure Appl. Math. 57 (2004), 987-1014.

[7] J. Colliander, M. Keel, G. Staffilani, H. Takaoka and T. Tao, Global well-posedness for the cubic nonlinear Schrödinger equation in $H^{s}\left(\mathbb{R}^{2}\right)$ for $s>\frac{1}{2}$, Discrete Contin. Dyn. Syst. 21 (2008), 665-686.

[8] J. Colliander, M. Keel, G. Staffilani, H. Takaoka and T. Tao, Global well-posedness and scattering for the energy-critical nonlinear schrödinger equation in $\mathbb{R}^{3}$, Ann. Math. 167 (2008), 767-865.

[9] J. Colliander and T. Roy, Bootstrapped Morawetz estimates and resonant decomposition for low regularity global solutions of cubic $N L S$ on $\mathbb{R}^{2}$, preprint, arXiv:0811.1803.

[10] J. Colliander, S. Raynor, C. Sulem, and J.D. Wright, Ground state mass concentration in the $L^{2}$-critical nonlinear Schödinger equation below $H^{1}$, Math. Res. Lett. 12 (2005), 357-375. 
[11] D. de Silva, N. Pavlovic, G. Staffillani, and N. Tzirakis, Global Well-Posedness for the $L^{2}$ critical nonlinear Schroedinger equation in higher dimensions, Commun. Pure. Appl. Anal. 6 (2007), 1023-1041.

[12] Y. Fang and M. Grillakis, On the global existence of rough solutions of the cubic defocusing Schrodinger equation in $\mathbb{R}^{2+1}$. J. Hyperbolic Differ. Equ. 4 (2007), 233-257.

[13] M. Keel and T. Tao, Endpoint Strichartz estimates, Amer. J. Math. 120 (1998), 955-980.

[14] R. Killip, T. Tao and M. Visan, The cubic nonlinear Schrodinger equation in two dimensions with radial data, to appear in J. Europ. Math. Soc.

[15] S. Keraani and A. Vargas, A smoothing property for the $L^{2}$-critical NLS equations and an application to blowup theory, to appear in Ann. I. H. Poincaré-AN(2008), doi:10.1016/j.anihpc.2008.03.001.

[16] S. Lee, Bilinear Restriction Estimates for Surfaces with Curvatures of Different Signs, Trans. Amer. Math. Soc. 358 (2006), 3511-3533.

[17] S. Lee and A. Vargas, Sharp null form estimates for the wave equation, Amer. J. Math. 130 (2008), 1279-1326.

[18] C. Miao, G. Xu and L. Zhao, Global well-posedness and scattering for the mass-critical Hartree equation with radial data, J. Math. Pure Appl. 91 (2009), 49-79.

[19] J. Peetre, New thoughts on Besov spaces, Duke University Matematicsl Series 1, Durham N. C. 1976.

[20] R. Strichartz, Restriction of Fourier transform to quadratic surfaces and decay of soluitons of wave equations, Duke Math. J. 44 (1977), 705-774.

[21] T. Tao, A Sharp bilinear restriction estimate for paraboloids, Geom. Funct. Anal. 13 (2003), 1359-1384.

[22] T. Tao, Nonlinear dispersive equations. Local and global analysis, CBMS 106, eds: AMS, 2006.

[23] T. Tao, A. Vargas, L. Vega, A bilinear approach to the restriction and Kakeya conjectures, J. Amer. Math. Soc. 11 (1998), 967-1000.

[24] T. Tao, M. Visan and X. Zhang, The Schrödinger equations with combined power-typ nonlinearities, Commun. PDE 32 (2007), 1281-1343.

[25] T. Wolff, A sharp bilinear cone restriction estimate, Ann. of Math. 153 (2001), 661-698.

Department of Applied Mathematics, Hankyong National University, Ansong 456-749, Republic of KoreA

E-mail address: mchae@hknu.ac.kr

Department of Mathematics, and Institute of Pure and Applied Mathematics, Chonbuk National University, Jeonju 561-756, Republic of Korea

E-mail address: changocho@chonbuk.ac.kr

Department of Mathematical Sciences, Seoul National University, Seoul 151747, Republic of Korea

E-mail address: shklee@snu.ac.kr 